\title{
Anais do III Simpósio de Trauma, Urgência e Emergência do Delta
}
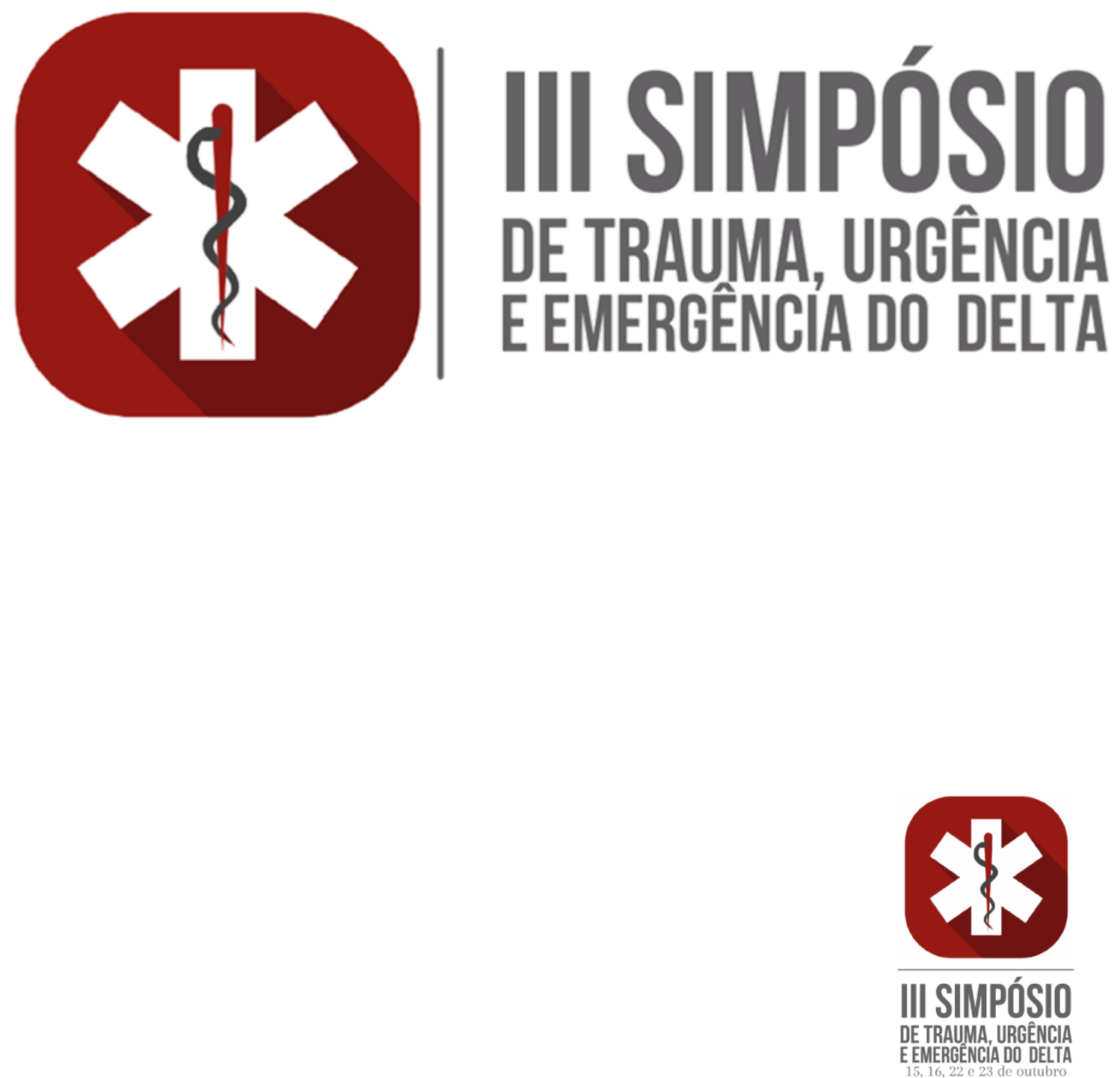

Parnaíba - PI 


\section{Ficha Técnica}

Evento: III Simpósio de Trauma, Urgência e Emergência do Delta

Organização: Liga Acadêmica de Trauma, Urgência e Emergência do Delta LATURE/UFDPar

Data/Período: 15, 16, 22 e 23 de outubro de 2021

Local: Edição on-line

Tema central: Urgências e Emergências em Saúde

Data das Apresentações dos Trabalhos Científicos: 22 e 23 de outubro

Formato: Temas Livres (Apresentação Oral) 


\section{Sumário}

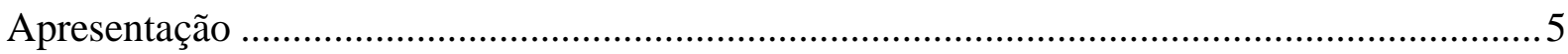

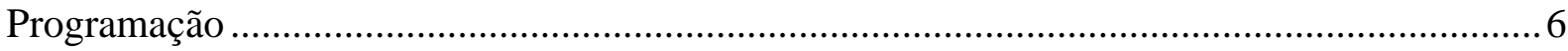

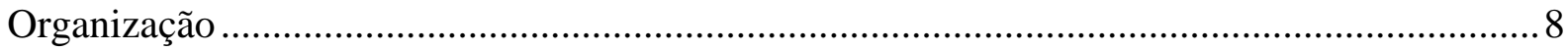

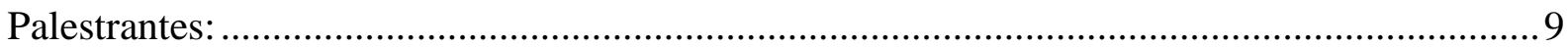

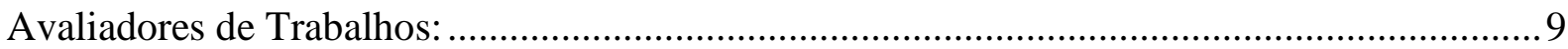

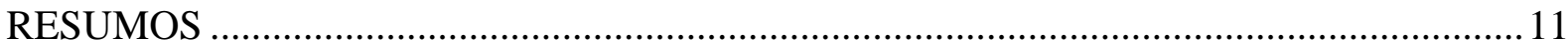

A IMPORTÂNCIA DA COMUNICAÇÃO EFETIVA NOS SERVIÇOS DE URGÊNCIA E EMERGÊNCIA: REVISÃO INTEGRATIVA DA LITERATURA ….................................... 12

A INFLUÊNCIA DO FLUXO SALIVAR NA SAÚDE ORAL …........................................ 13

ACESSO VASCULAR POR VIA INTRAÓSSEA COMO ALTERNATIVA EM

EMERGÊNCIA

ACHADOS CLÍNICOS DE IMPORTÂNCIA ODONTOLÓGICA CAUSADOS POR

VIOLÊNCIA INFANTIL DURANTE A PANDEMIA DE COVID-19.

MORTALIDADE POR CÂNCER COLORRETAL: COMPARATIVO ENTRE AS

REGIÕES BRASILEIRAS

SÍFILIS CONGÊNITA: UMA ANÁLISE DO PERFIL EPIDEMIOLÓGICO DA CIDADE DE PARNAÍBA EM COMPARAÇÃO AO CENÁRIO NO ESTADO DO PIAUÍ E

NACIONAL ENTRE 2014 A 2018.

ANÁLISE EPIDEMIOLÓGICA DOS CASOS NOTIFICADOS DE MENINGITE NA

CIDADE DE TERESINA ENTRE OS ANOS DE 2015 E 2020.

ESTUDO COMPARATIVO DO NÚMERO DE AVALIAÇÕES ANTROPOMÉTRICAS REALIZADAS EM PARNAÍBA, NO PIAUÍ E NO NORDESTE ENTRE 2015 E 2020...... 19

ASSISTÊNCIA DE ENFERMAGEM FRENTE AO PACIENTE POLITRAUMATIZADO EM EMERGÊNCIA HOSPITALAR: UMA REVISÃO DA LITERATURA ........................ 20

CRISE ANAFILÁTICA: MANEJO NA EMERGÊNCIA MÉDICA .....................................21

DESAFIOS DAS PESSOAS QUE VIVEM COM HIV/AIDS FRENTE À PANDEMIA DE COVID-19: UMA REVISÃO INTEGRATIVA

ESTUDO COMPARATIVO DO PERFIL DE MORTALIDADE POR DOENÇAS

CARDIOVASCULARES ENTRE A POPULAÇÃO DO BRASIL E DO PIAUÍ . .23

ANÁLISE EPIDEMIOLÓGICA DAS INTERNAÇÕES DE URGÊNCIA POR FRATURA DO FÊMUR NO PIAUÍ NO PERÍODO DE 2011 A 2020

ANÁLISE EPIDEMIOLÓGICA DOS CASOS DE HANSENÍASE NO PIAUÍ NOS ANOS

DE 2015 A 2019.

ANÁLISE EPIDEMIOLÓGICA DOS CASOS DE INFARTO AGUDO DO MIOCÁRDIO NO PIAUÍ: UM RECORTE DO PERÍODO DE 2015 A 2020.

ANÁLISE EPIDEMIOLÓGICA DAS INTERNAÇÕES E DOS ÓBITOS POR

INSUFICIÊNCIA RENAL NO BRASIL ENTRE OS ANOS DE 2010 E 2020. 
ANÁLISE EPIDEMIOLÓGICA COMPARATIVA ENTRE O NÚMERO DE INTERNAÇÕES E ÓBITOS POR NEOPLASIA MALIGNA DA MAMA, NA REGIÃO DA PLANÍCIE LITORÂNEA, NO PERÍODO DE 2015 À 2020.

INTUBAÇÃO SUBMENTONIANA PARA MANEJO DAS VIAS AÉREAS EM PACIENTES COM FRATURAS COMPLEXAS DE FACE.

MANEJO E ACESSO ÀS VIAS AÉREAS DE PACIENTES COM TRAUMA DE FACE EM SITUAÇÕES DE EMERGÊNCIA.

MANIFESTAÇÕES CLÍNICAS DA COVID-19 EM GESTANTES: UMA REVISÃO SISTEMÁTICA.

ANÁLISE EPIDEMIOLÓGICA DA MORTALIDADE MATERNA POR ECLÂMPSIA NO

ESTADO DO PIAUÍ ENTRE OS ANOS DE 2015 E 2019

INTERNAÇÕES E TAXA DE MORTALIDADE POR NEOPLASIA MALIGNA DE PRÓSTATA NO PIAUÍ DE 2016 A 2020.

O ADOECIMENTO E OS PROCESSOS DE LUTO NO CONTEXTO DE PANDEMIA DE COVID-19: UMA REVISÃO INTEGRATIVA

MANIFESTAÇÕES CLÍNICAS, DIAGNÓSTICO E TRATAMENTO PARA A

PARALISIA DE BELL

PARTICULARIDADES DO TRAUMA BUCO MAXILO FACIAL QUE DIFICULTAM O ATENDIMENTO DE EMERGÊNCIA.

ANÁLISE DA PREVALÊNCIA DE HIPERTENSÃO ARTERIAL SISTÊMICA EM CRIANÇAS E ADOLESCENTES: REVISÃO INTEGRATIVA

PRINCIPAIS IMPLICAÇÕES DO TRATAMENTO ODONTOLÓGICO EM PACIENTES COM SÍNDROME DE MOEBIUS

PROCEDIMENTOS E CUSTOS HOSPITALARES ACERCA DA REALIZAÇÃO DE PAROTIDECTOMIA PARCIAL OU SUBTOTAL NO ÂMBITO DO SISTEMA ÚNICO DE SAÚDE NA REGIÃO NORDESTE NO PERÍODO DE 2011 A 2019.

IMPACTO DA PANDEMIA NO RASTREIO DO CÂNCER DE MAMA NO ESTADO DO PIAUÍ.

REPOSIÇÃO VOLÊMICA EM PACIENTES VÍTIMAS DE TRAUMA: UMA REVISÃO DE LITERATURA.

PADRÃO ESPACIAL E TEMPORAL DOS ÓBITOS POR AIDS NO ESTADO DO MARANHÃO NO PERÍODO DE 2011 A 2019

REPERCUSSÕES CLÍNICO-OBSTÉTRICAS DE GESTANTES COM COVID-19: UMA REVISÃO SISTEMÁTICA

A SISTEMÁTICA MULTIDISCIPLINAR NO ATENDIMENTO DE EMERGÊNCIA EM PACIENTES VÍTIMAS DE TRAUMAS FACIAIS.

UM CONTRAPONTO ENTRE A INTERVENÇÃO CIRÚRGICA E A CONSERVADORA NO TRAUMA HEPÁTICO

TROMBOEMBOLISMO PULMONAR BILATERAL: RELATO DE CASO DE UM HOSPITAL TERCIÁRIO DE FORTALEZA-CE. 
TRATAMENTO CIRÚRGICO DE INVERSÃO UTERINA AGUDA PÓS PARTO: UM ESTUDO COMPARATIVO ENTRE PIAUÍ, NORDESTE E BRASIL.

TRAUMATISMO DURANTE O PARTO NO NEONATO: UMA ANÁLISE

EPIDEMIOLÓGICA COMPARATIVA EM NÍVEL NACIONAL, REGIONAL E

ESTADUAL

VANTAGENS E DESVANTAGENS DO USO DE ÁCIDO HIALURÔNICO NO PREENCHIMENTO ESTÉTICO DE OLHEIRAS 


\section{Apresentação}

O Simpósio de Trauma, Urgência e Emergência do Delta - SITUE é um evento realizado pela Liga Acadêmica de Trauma, Urgência e Emergência do Delta - LATURE, que visa à promoção de um amplo debate acerca de temáticas pertinentes e desafiadoras no campo de atendimento em Urgência e Emergência, contribuindo para a formação de profissionais qualificados na área. Após o sucesso das duas primeiras edições do SITUE e diante da impossibilidade de realização do evento de forma presencial pelo contexto sanitário vivido atualmente, o III SITUE surge com uma proposta ainda mais inovadora e relevante. O objetivo do evento se expande e busca elevar os padrões dos eventos acadêmicos desenvolvidos na cidade de Parnaíba, Piauí, ao ampliar o acesso ao evento em seu formato online, bem como a participação de palestrantes de renome a nível nacional. Em sua terceira edição, o SITUE buscou abranger, ainda mais, temas multidisciplinares, de modo a englobar cursos para além da Medicina. Nesse sentido, utilizou-se como metodologia a realização de palestras com as mais novas atualizações publicadas sobre a temática central do evento, ministradas por profissionais com reconhecido conhecimento acerca dos assuntos abordados, como enfermeiros, médicos e fisioterapeutas. Ademais, o evento contou com Edital para Submissão de Trabalhos Científicos, na modalidade Tema livre oral, de forma a estimular a pesquisa acadêmica. Desse modo, o objetivo do III SITUE foi ampliar o acesso às informações e conhecimentos científicos nas diversas áreas da Urgência e Emergência, esse campo tão emergente e atual. 


\section{Programação}

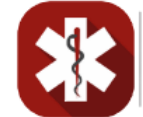

III SIMPÓSIO

DE TRAUMA. URGÊNCIA

EMERGENCIA DO DELTA

\section{5/10}

18H - DEODATO NARCISO DE OLIVEIRA

CONDUTA EM EMERGÊNCIAS ORTOPÉDICAS

19H - RURION MENESES

ABORDAGEM DO ABDOME AGUDO NO PRONTO SOCORRO

2OH - NAGELE DE SOUSA LIMA

MANEJO DO INICIAL DO PACIENTE EM CHOQUE

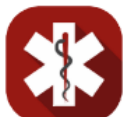

III SIMPÓSIO

DE TRAUMA, URGÊNCIA

EEMERGENCIA DO DELTA

\section{2/10}

$18 \mathrm{H}$ - BRENO DOUGLAS

CONDUTA NA INSUFICEENNCIA RESPIRATÓRIA NO PRONTO SOCORRO

19H - IRENE MEDEIROS

VENTILAÇ̃̃O MECÂNICA INVASIVA NA EMERGÊNCIA

2OH - WESLEY PINTO

CUIDADOS PÓS PARADA

CARDIORRESPIRATÓRIA PELO ENFERMEIRO

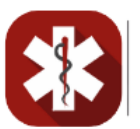

III SIMPÓSIO

DE TRAUMA, URGENCIA

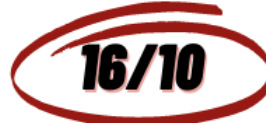

18H - JULE R O G SANTOS

REANIMAÇÃO CARDIOPULMONAR:

O QUE OS LIVROS NÃO ENSINAM

$19 \mathrm{H}$ - FREDERICO ARNAUD

INTUBAÇ̃̃O OROTRAQUEAL DE SEQUÊNCIA

RÁPIDA: O DESAFIO DA PRÁTICA

2OH - PATRÍCIA MELLO

CETOACIDOSE DIABÉTICA: DO

DIAGNÓSTICO AO TRATAMENTO

III SIMPÓSIO

EE TRAUMA, URGENCIA

EEMERGENCIA DO DELT

\section{3/10}

18H - KHALIL FEITOSA

CHOQUE SÉPTICO NO PRONTO

SOCORRO

19H - JOBERT MITSON

MANEJO INICIAL DO INFARTO AGUDO DO

MIOCÁRDIO

2OH - EUGÊNIO FRANCO

INTUBAÇ̃̃O OROTRAQUEAL NA

EMERGÊNCIAO 


\begin{tabular}{|c|c|c|c|c|}
\hline Data/hora & $15 / 10$ & $16 / 10$ & $22 / 10$ & $23 / 10$ \\
\hline 18:00h & $\begin{array}{c}\text { Deodato Narciso - } \\
\text { Conduta em } \\
\text { emergências } \\
\text { ortopédicas }\end{array}$ & $\begin{array}{c}\text { Jule Santos - } \\
\text { Reanimação } \\
\text { cardiopulmonar: o } \\
\text { que os livros não } \\
\text { ensinam }\end{array}$ & $\begin{array}{l}\text { Breno Douglas - } \\
\text { Conduta na } \\
\text { insuficiência } \\
\text { respiratória no Pronto } \\
\text { Socorro }\end{array}$ & $\begin{array}{l}\text { Khalil Feitosa - } \\
\text { Choque séptico no } \\
\text { Pronto Socorro }\end{array}$ \\
\hline 19:00h & $\begin{array}{l}\text { Rurion Meneses - } \\
\text { Abordagem do } \\
\text { abdome agudo no } \\
\text { Pronto Socorro }\end{array}$ & $\begin{array}{l}\text { Frederico Arnaud - } \\
\text { Intubação } \\
\text { orotraqueal de } \\
\text { sequência rápida: o } \\
\text { desafio da prática }\end{array}$ & $\begin{array}{c}\text { Irene Medeiros - } \\
\text { Ventilação mecânica } \\
\text { invasiva na } \\
\text { emergência }\end{array}$ & $\begin{array}{c}\text { Jobert Mitson - } \\
\text { Manejo inicial do } \\
\text { Infarto Agudo do } \\
\text { Miocárdio }\end{array}$ \\
\hline 20:00h & $\begin{array}{l}\text { Nagele Lima - } \\
\text { Manejo do inicial } \\
\text { do paciente em } \\
\text { choque }\end{array}$ & $\begin{array}{l}\text { Patrícia Mello - } \\
\text { Cetoacidose } \\
\text { diabética: do } \\
\text { diagnóstico ao } \\
\text { tratamento }\end{array}$ & $\begin{array}{l}\text { Wesley Pinto - } \\
\text { Cuidados pós-parada } \\
\text { cardiorrespiratória } \\
\text { pelo enfermeiro }\end{array}$ & $\begin{array}{c}\text { Eugênio Franco - } \\
\text { Intubação } \\
\text { orotraqueal na } \\
\text { emergência }\end{array}$ \\
\hline
\end{tabular}




\section{Organização}

\section{Coordenação:}

Deodato Narciso de Oliveira Castro Neto (Coordenador Geral)

Ivan Rodrigues Silva (Sub-Coordenador)

Érica de Araújo Silva Mendes (Sub-Coordenadora)

\section{Diretoria:}

Natalya de Carvalho Lima (Presidente)

Igor dos Santos Cavalcante (Vice-Presidente)

Pedro Henrique dos Santos Silva (Diretor de Pesquisa)

Isabella Pires Gomes Mendes (Diretora de Extensão)

Victor Trindade da Cruz (Diretor de Ensino)

Cláudio Vinícius Barroso Queirós de Lima (Secretário geral)

\section{Comissão de Programação:}

Cláudio Vinícius Barroso Queirós de Lima

Ingrid Brandão Cardoso Paz

Isabella Pires Gomes Mendes

Natalya de Carvalho Lima

\section{Comissão de Pesquisa:}

Evandro Cavassani Gimenes

Igor dos Santos Cavalcante

Pedro Henrique dos Santos Silva

Paulo Cesar Monteiro Florêncio

\section{Comissão de Logística:}

João Victor Carvalho Barbosa

Victor Trindade da Cruz

Sarah da Conceição t'Lam 


\section{Comissão de Marketing:}

Luana Mazza Malta

Sofia Carneiro da Cunha

José Freire Furtado Neto

\section{Comissão de Patrocínio:}

Julliana Emily Matos e Silva

Lady Jane da Silva Macedo

Lucas de Carvalho Techi

\section{Palestrantes:}

Ana Irene Carlos de Medeiros

Breno Douglas Dantas Oliveira

Deodato Narciso de Oliveira Castro Neto

Eugênio Santana Franco Filho

Frederico Carlos de Sousa Arnaud

Jobert Mitson Silva dos Santos

Jule Rouse de Oliveira Gonçalves Santos

Khalil Feitosa de Oliveira

Nagele de Sousa Lima

Patrícia M. Veiga de Carvalho Mello

Rurion Charles de Souza Meneses

Wesley Pinto da Silva

Avaliadores de Trabalhos:

Anderson de Sousa Escórcio

Andressa Araujo Rodrigues da Silva

Andressa Rios Frota

Carliane da Conceição Machado Sousa 
Franciele Basso Fernandes Silva

Hélio Mateus Silva Nascimento

Juliana Félix de Melo

Luciana Rocha Faustino

Maria Gislene Santos Silva

Renata Paula Lima Beltrão

Sara Sabrina Vieira Cirilo 


\section{RESUMOS}




\title{
A IMPORTÂNCIA DA COMUNICAÇÃO EFETIVA NOS SERVIÇOS DE URGÊNCIA E EMERGÊNCIA: REVISÃ̃ INTEGRATIVA DA LITERATURA
}

\author{
Lara Beatriz de Sousa Araújo ${ }^{1}$, Emerson Galdino Rodrigues dos Santos ${ }^{2}$, Rafaela de Jesus \\ Portugal $^{3}$, Mayara Aline de Barros Lima ${ }^{4}$, Caroline Taiane Santos da Silva ${ }^{5}$
}

Introdução: A comunicação é um importante instrumento de interação e transmissão de mensagens, podendo ou não ocorrer verbalmente. Entretanto, o atendimento hospitalar em contexto de urgência e emergência é caracterizado pela celeridade, o que pode ocasionar problemas na comunicação, podendo representar perigo aos pacientes. Objetivo: Discutir acerca da comunicação efetiva nos serviços de urgência e emergência. Método: Revisão integrativa, realizada através das bases SciELO, LILACS e MEDLINE, pelos descritores: "Socorro de Urgência", "Barreiras de Comunicação" e "Comunicação", combinados pelo booleano AND. Foram incluídos artigos online, em português, espanhol e inglês, dos últimos cinco anos. Foram excluídos: literatura cinzenta e estudos repetidos nas bases. Dessa forma, foram encontrados 15 estudos. Resultados: Os problemas de comunicação podem se dar por diversos motivos, como idiomas diferentes, dificuldades em expressar ou compreender informações de maneira efetiva. No entanto, em emergências, os problemas de comunicação podem ter consequências perigosas para os pacientes, uma vez que o risco de mortalidade é maior e a passagem de informações de forma errônea pode colocar vidas em perigo. Nesse sentido, se faz necessário a adoção de tecnologias de saúde, como aplicativos que permitem traduzir idiomas, além de práticas em grupo, voltados ao treinamento de comunicação com a equipe multidisciplinar, a fim de diminuir os riscos. Conclusão: A comunicação é um significativo método de expressão, sendo de sumo importância que ocorra de maneira adequada. Dessa forma, a comunicação entre profissional, paciente, família ou entre a equipe precisa ocorrer de maneira efetiva, para uma boa compreensão e redução do perigo.

Descritores: Socorro de Urgência; Barreiras de Comunicação; Comunicação.

\footnotetext{
${ }^{1}$ Universidade Federal do Piauí (UFPI); Teresina, Piauí, Brasil; larabeatriz@ufpi.edu.br; 0000-0003-0820-203X

${ }^{2}$ Centro Universitário de Tecnologia e Ciências (UNIFTC); Jequié, Bahia, Brasil; galdinoegrs@ gmail.com; 0000-0003-2290-4759

${ }^{3}$ Universidade Salvador (UNIFACS); Salvador, Bahia, Brasil; rafaelaenfermagem2@ gmail.com; 0000-00027895-9372

${ }^{4}$ Universidade Norte do Paraná (UNOPAR); Garanhuns, Pernambuco, Brasil; alinnebarros1@gmail.com; 0000-0002-9205-2072

${ }^{5}$ Escola Bahiana de Medicina e Saúde Pública (EBMSP); Salvador, Bahia, Brasil; carolinetaiane.enfa@gmail.com; 0000-0001-9471-6760
} 


\title{
A INFLUÊNCIA DO FLUXO SALIVAR NA SAÚDE ORAL
}

\author{
Rafaela Inês da Silva ${ }^{1}$, Daniela Maria Santos Falcão ${ }^{2}$, Carina Scanoni Maia ${ }^{3}$.
}

Introdução: A saliva é um líquido incolor que é produzido pelas glândulas salivares maiores e menores, composta, principalmente, por água. Sua menor parte é composta por proteínas e íons. Ela tem a função de lubrificação da cavidade oral, permitindo, assim, a neutralização dos ácidos, a deglutição, fonação, digestão e é bactericida. Objetivo: O objetivo desse trabalho é identificar o papel que o fluxo salivar tem na manutenção da saúde oral. Métodos: Realizou-se uma revisão de literatura selecionando artigos que tratassem sobre algumas das doenças que são influenciadas pela alteração do fluxo salivar. Resultados: O fluxo salivar é dividido em estimulado e o não-estimulado, essa divisão ocorre devido a quantidade de estímulos que a saliva recebe durante o dia ou durante a noite. Quando a saliva está em repouso, sem estímulos, geralmente influenciados pela alimentação, ela tem seu fluxo e o potencial hidrogeniônico dentro do padrão normal, mas quando ela recebe componentes exógenos, como ácidos, doces, salgados e amargos os estímulos produzem um aumento do fluxo salivar e passa a ser considerada estimulada. Nota-se, também, que o excesso da produção de saliva, bem como a diminuição de produção pode gerar consequências para a saúde oral. Porque a saliva possui a capacidade tampão, com isso, quando o fluxo salivar está abaixo do normal, aumenta o potencial hidrogeniônico e quando o fluxo está acima, diminui o potencial hidrogeniônico. Essas mudanças no fluxo salivar influência no desenvolvimento de doenças, como nas doenças periodontais, no desenvolvimento de cáries dentárias e também na xerostomia "boca seca". Conclusão: Conclui-se que o estudo permite reforçar sobre a importância do fluxo salivar manter seu padrão, para, assim, não desenvolver doenças na cavidade oral.

Descritores: Saliva; Saúde Bucal; Cárie Dentária; Xerostomia.

\footnotetext{
${ }^{1}$ Discente de Odontologia da Universidade Federal de Pernambuco. Recife, Pernambuco, Brasil. E-mail: rafinhainess198@gmail.com

${ }^{2}$ Discente de Odontologia da Universidade Federal de Pernambuco. Recife, Pernambuco, Brasil. E-mail: danielamsfalcao@gmail.com

${ }^{3}$ Professora de Histologia e Embriologia Oral da Universidade de Pernambuco. Recife, Pernambuco, Brasil. Email: carina.maia@ufpe.br
} 


\title{
ACESSO VASCULAR POR VIA INTRAÓSSEA COMO ALTERNATIVA EM EMERGÊNCIA
}

\author{
Francisco Lucas Leandro de Sousa ${ }^{1}$, Danilo da Silva Alves ${ }^{2}$
}

Introdução: A punção intraóssea consiste na introdução de uma agulha com o intuito de ter acesso à circulação sistêmica. Esse procedimento é bastante utilizado em pediatria principalmente em situações de emergência. Objetivos: Descrever a importância do acesso intraósseo nas situações de emergência. Métodos: Refere-se a um estudo de revisão integrativa, realizada a partir de levantamentos bibliográficos nas bases de dados LILACS e SCIELO com o auxílio dos descritores: "Infusão Intraóssea", "Emergências" e "Métodos". Como critérios de inclusão artigos originais que abordassem a temática, disponíveis online, na íntegra, nos idiomas português e inglês, entre 2017 e 2021 e como critérios de exclusão: estudos repetidos nas bases de dados. Totalizando 14 estudos para compor a revisão. Resultados: Estudos enfatizam que o acesso intraósseo se mostrou uma técnica bastante eficaz, segura e satisfatória caracterizando-se como uma alternativa a mais para estabilização do quadro do paciente. Vale enfatizar que esse procedimento é bastante comum, utilizado na maioria das vezes em pacientes que não dispõe de acesso venoso eficaz em decorrência do seu estado clínico de saúde. Esse procedimento, oferece duração de 24 horas, além de ser usado para infusão de medicamentos e coleta de material biológico. Desse modo, a via intraóssea consolida-se, como uma segunda opção de acesso vascular no atendimento a emergências, por apresentar rápida e fácil execução, com o mínimo de complicações para o paciente. Conclusão: Nota-se que essa técnica de acesso intraósseo necessita ser mais difundida pelos profissionais, por apresentar-se como uma alternativa eficaz para os pacientes.

Descritores: Infusões intraósseas; Emergências; Métodos.

\footnotetext{
${ }^{1}$ Centro Universitário Maurício de Nassau (UNINASSAU), Fortaleza, Ceará, Brasil. E-MAIL: lucasleandro2912@gmail.com. ORCID: https://orcid.org/0000-0003-2802-2378

${ }^{2}$ Centro Universitário Maurício de Nassau (UNINASSAU), Fortaleza, Ceará, Brasil. E-MAIL: enferdanilo.alves@gmail.com. ORCID: https://orcid.org/000-0001-7663-223X
} 


\section{ACHADOS CLÍNICOS DE IMPORTÂNCIA ODONTOLÓGICA CAUSADOS POR VIOLÊNCIA INFANTIL DURANTE A PANDEMIA DE COVID-19}

Ana Júlia Gomes Leal ${ }^{1}$; Anderson Marlon Albino de Abreu ${ }^{2}$; Nayanne Gomes Araújo ${ }^{3}$; José Fernando Marinho de Almeida Moreira ${ }^{4}$; Héberte Santana Arruda ${ }^{5}$.

Introdução: Durante a pandemia e o isolamento social, foi registrado um grande aumento no número de casos de violência física, sexual e psicológica contra crianças e adolescentes. Tal episódio acontece onde menos se espera, em casa, e através de pessoas próximas que convivem diariamente com esses menores. O fechamento de escolas e creches durante o período pandêmico foi um dos principais fatores que agravaram esse alto índice de violência. Objetivo: Apresentar os principais achados clínicos de importância odontológica, decorrentes da violência infantil durante a pandemia do COVID-19. Métodos: Foi realizada uma seleção de artigos relacionados ao tema abordado nas bases de dados PubMed, BVS e SciELO. Utilizaram-se como descritores: Maus-Tratos Infantis, Assistência Odontológica para Crianças e COVID-19. Foram aplicados critérios de inclusão e exclusão, sendo selecionados 10 artigos que estão presentes nessa revisão. Resultados: Dentre os principais achados orofaciais decorrentes de abuso infantil, encontramos: contusões na face, pescoço e estruturas periorais; traumas ósseos e dentários; queimaduras; hematomas; marcas de mordida; além de manifestações bucais de doenças sexualmente transmissíveis como a sífilis, herpes e gonorreia. Conclusão: Compete ao cirurgião-dentista o conhecimento necessário para uma eficiente atuação nos casos de violência infantil. A correta identificação da etiologia dos achados clínicos é imprescindível para a segurança da vítima e consequentemente para que haja a devida denúncia do agressor.

Descritores: Maus-Tratos Infantis; Assistência Odontológica para Crianças; COVID-19.

\footnotetext{
${ }^{1}$ Universidade Federal de Pernambuco - UFPE - Recife-PE, Graduanda de Odontologia, $6^{\circ}$ período, lealgomesjulia@hotmail.com

${ }^{2}$ UFPE - Recife-PE, Graduando de Odontologia, 6º período, andersonabreu2012@gmail.com

${ }^{3}$ UFPE - Recife-PE, Graduanda de Odontologia, $6^{\circ}$ período, gomesnayanne7@gmail.com

${ }^{4}$ UFPE - Recife-PE, Graduanda de Odontologia, $6^{\circ}$ período, fernandinhobrawli@ hotmail.com

${ }^{5}$ Faculdade de Odontologia de Pernambuco - UPE - Recife-PE, Prof. MSc do Curso de Odontologia do Centro Universitário Brasileiro - Unibra, hebertearruda@gmail.com
} 


\section{MORTALIDADE POR CÂNCER COLORRETAL: COMPARATIVO ENTRE AS REGIÕES BRASILEIRAS}

Vinicius Souza Freires ${ }^{1}$; Maria Beatriz Pereira de Paula Rocha²; Andressa Carvalho Pereira ${ }^{3}$; Rafael Santos Correia ${ }^{4}$; Franciele Basso Fernandes Silva ${ }^{5}$.

Introdução: O câncer colorretal (CCR) é o terceiro tipo de câncer com maior mortalidade no Brasil. As diferenças culturais e socioeconômicas evidenciadas entre as regiões brasileiras podem propiciar diferentes taxas de mortalidade por esse agravo. Objetivo: Analisar as taxas de mortalidade por CCR entre as regiões do Brasil, durante o ano de 2019. Métodos: Trata-se de um estudo epidemiológico com dados coletados no Sistema de Informações Hospitalares do SUS (SIH/SUS), relativos aos óbitos por câncer colorretal em 2019 no Brasil, por região. As variáveis avaliadas foram sexo e faixa etária. Resultados: No ano de 2019, o número total de óbitos por CCR no Brasil foi de 20.578, o que corresponde a uma taxa de mortalidade (TM) de 9,76 por cem mil habitantes. Dentre as regiões, destacam-se com maior e menor TM, as regiões Sudeste $(12,68 / 100$ mil habitantes) e Norte (3,78/100 mil habitantes), respectivamente. Em relação à faixa etária, notou-se maior número de casos entre pessoas de 60 a 69 anos, com 25,67\% dos óbitos (5.283 casos). Todavia, a maior TM foi encontrada em pacientes acima dos 80 anos (60,131/100 mil habitantes). Entre os sexos houve semelhança nas taxas de mortalidade, sendo 10,03 para os homens e 9,51 para mulheres. Conclusão: O perfil de mortalidade por CCR se mostra relacionado a idades mais avançadas e não houve diferença significativa entre os sexos. O Sudeste, região com maior TM, possui índices socioeconômicos e de saúde mais elevados, o que poderia favorecer maior acesso a métodos diagnósticos e notificações mais eficientes.

Descritores: Mortalidade, Neoplasias Colorretais, Epidemiologia.

\footnotetext{
${ }^{1}$ Universidade Federal do Delta do Parnaíba (UFDPar), Parnaíba, Piauí. E-mail: vinifreires@gmail.com

${ }^{2}$ Instituto de Educação Superior do Vale do Parnaíba (IESVAP) Parnaíba, Piauí. E-mail: mariabeatriz_rocha@hotmail.com

${ }^{3}$ Universidade Federal do Delta do Parnaíba (UFDPar), Parnaíba, Piauí. E-mail: andressa_carvalho@ufpi.edu.br, https://orcid.org/0000-0001-7721-0661

${ }^{4}$ Universidade Federal do Delta do Parnaíba (UFDPar), Parnaíba, Piauí. E-mail: rafael0094@gmail.com

${ }^{5}$ Universidade Federal do Delta do Parnaíba (UFDPar), Parnaíba, Piauí. E-mail: francibasso2@ hotmail.com
} 


\section{SÍFILIS CONGÊNITA: UMA ANÁLISE DO PERFIL EPIDEMIOLÓGICO DA CIDADE DE PARNAÍBA EM COMPARAÇÃO AO CENÁRIO NO ESTADO DO PIAUÍ E NACIONAL ENTRE 2014 A 2018}

Livia Rocha Santos ${ }^{1}$; Martha Laura Leão dos Santos Silva²; Igor dos Santos Cavalcante ${ }^{3}$; Ana Vitória Meireles Veiga ${ }^{4}$; Caroline Camargo Bandeira da Silveira Luz ${ }^{5}$

Introdução: A sífilis congênita é transmitida via placenta ou durante o parto, quando não é tratada ou tratada da forma inadequada pode levar a morte fetal e/ou neonatal entre outras complicações, sendo importante a atuação da rede de assistência ao pré-natal para o diagnóstico e tratamento precoce das gestantes. Objetivo: Comparar o perfil epidemiológico dos casos de sífilis congênita do município de Parnaíba entre 2014 e 2018 e compará-los ao cenário estadual e nacional. Métodos: Estudo epidemiológico descritivo-quantitativo. Os dados foram coletados do Departamento de Informática do Sistema Único Saúde (DATASUS), baseado no Sistema de Informação de Agravos de Notificação (SINAN). As variáveis analisadas foram número total de casos de sífilis congênita e sífilis materna, escolaridade, realização do pré-natal, momento do diagnóstico, classificação final e evolução do caso notificado. Os dados foram tabulados para análise. Discussão: Durante o período analisado, foram notificados 107.574 casos de sífilis congênita no Brasil, sendo 1.782 casos no Piauí, dos quais 209 (11,72\%) eram referentes à cidade de Parnaíba. No cenário nacional, estadual e municipal, na maioria dos casos as gestantes apresentavam ensino médio incompleto, o acompanhamento pré-natal foi realizado e o diagnóstico de sífilis materna ocorreu nesse período, a evolução foi crianças vivas diagnosticadas com sífilis congênita recente. Conclusão: A cidade de Parnaíba apresenta um perfil epidemiológico semelhante ao observado no Estado do Piauí e no Brasil. Incluindo o crescimento no número de casos ao longo do período analisado, por possíveis fragilidades no acesso, diagnóstico e tratamento da doença no pré-natal.

Descritores: Epidemiologia; Sífilis Congênita; Sífilis.

\footnotetext{
${ }^{1}$ Universidade Federal do Delta do Parnaíba (UFDPar), Parnaíba, Piauí. E-mail: liviarocha063@gmail.com

${ }^{2}$ Universidade Federal do Delta do Parnaíba (UFDPar), Parnaíba, Piauí. E-mail: marthalaura928@gmail.com

${ }^{3}$ Universidade Federal do Delta do Parnaíba (UFDPar), Parnaíba, Piauí. E-mail: igorsc@live.com

${ }^{4}$ Universidade Federal do Delta do Parnaíba (UFDPar), Parnaíba, Piauí. E-mail: vitmei@ hotmail.com

${ }^{5}$ Universidade Federal do Delta do Parnaíba (UFDPar), Parnaíba, Piauí. E-mail:

dracarolinesilveira@outlook.com
} 


\section{ANÁLISE EPIDEMIOLÓGICA DOS CASOS NOTIFICADOS DE MENINGITE NA CIDADE DE TERESINA ENTRE OS ANOS DE 2015 E 2020}

Arícia Gomes Miranda ${ }^{1}$; Mikaelly Melgaço Nunes ${ }^{2}$; Carlos Antônio Soares de Sousa ${ }^{3}$; Ingrid Brandão Cardoso Paz ${ }^{4}$ Ivan Rodrigues Silva ${ }^{5}$.

Introdução: A meningite é uma doença infecciosa, causada por vírus ou bactéria, de notificação compulsória no Brasil. A doença gera a inflamação das meninges, membranas protetoras do cérebro e da medula espinhal e sua transmissão ocorre, sobretudo, por gotículas de saliva através do ar, sendo a melhor forma de prevenção a vacina. Nesse sentido, é relevante avaliar os casos de meningite, para que medidas de saúde pública possam ser direcionadas à manutenção do controle da doença. Objetivo: Analisar a epidemiologia dos casos de meningite notificados na cidade de Teresina entre os anos de 2015 e 2020. Métodos: Trata-se de um estudo epidemiológico, quantitativo, observacional e transversal dos casos notificados de meningite na cidade de Teresina, do Piauí, entre os anos de 2015 a 2020. As variáveis utilizadas foram: sexo e faixa etária. Os dados foram extraídos do Sistema Nacional de Informação sobre Doenças e Agravos de Notificação (DATASUS). Resultados: Durante o período analisado, houveram 820 casos notificados de meningite em Teresina. Houve uma prevalência do sexo masculino $60 \%(\mathrm{~N}=495)$ e da faixa etária de 20 a 39 anos com $30 \%$ dos casos $(\mathrm{N}=247)$. Crianças com menos de 5 anos representaram 17,4\% ( $\mathrm{N}=143)$ dos casos. Conclusão: Assim, houve uma prevalência de homens, entre 20 e 39 anos, entre notificações de meningite. Ademais, considerando que as crianças com menos de 5 anos são um grupo de risco para a doença, e representaram um percentual significativo, políticas públicas devem ser direcionadas para esse público, incentivando a sua vacinação.

Descritores: Epidemiologia; Meningite; Notificação de Doenças.

\footnotetext{
${ }^{1}$ Discente da Universidade Federal do Delta do Parnaíba. Email: ariciamiranda05@ gmail.com

${ }^{2}$ Discente da Universidade Federal do Delta do Parnaíba. Email: mikaellymel123@ gmail.com

${ }^{3}$ Discente da Universidade Federal do Delta do Parnaíba. Email: carlos17041@ gmail.com

${ }^{4}$ Discente da Universidade Federal do Delta do Parnaíba. Email: ingridbrandaoc@ gmail.com

${ }^{5}$ Docente da Universidade Federal do Delta do Parnaíba. Email: ivandoctor@gmail.com
} 


\section{ESTUDO COMPARATIVO DO NÚMERO DE AVALIAÇÕES ANTROPOMÉTRICAS REALIZADAS EM PARNAÍBA, NO PIAUÍ E NO NORDESTE ENTRE 2015 E 2020}

Breno Vítor Rodrigues Coqueiro Santana ${ }^{1}$, Isabella Pires Gomes Mendes², Francisco Nogueira do Rego $\mathrm{Neto}^{3}$, Priscylla Frazão Rodrigues ${ }^{4}$, Érica de Araújo Silva Mendes ${ }^{5}$

Introdução: Avaliação antropométrica (AA) representa a medida e análise de indicadores corporais, através da qual é possível escolher melhor conduta terapêutica e acompanhar mais eficientemente a evolução do paciente, garantindo melhor cuidado. Objetivo: Comparar o número de AA realizadas em Parnaíba, Piauí e Nordeste entre 2015 e 2020. Métodos: Estudo descritivo comparativo em que foram observados os números de AA realizadas por localidade e anos estudados. Todos os dados foram retirados do Sistema de Informações Ambulatoriais do SUS (SIA/SUS), no DATASUS. Resultados: Entre 2015 e 2017, foram realizados 28.950, 27.040 e 36.155 AA, respectivamente, em Parnaíba, totalizando 92.145, enquanto que entre 2018 e 2020 não foram encontrados registros. No Piauí, foram realizadas em todo o período, respectivamente, $1.352 .435,1.279 .640,1.454 .404,492.711,73.503$ e 19.848 AA, totalizando 4.086.501. No Nordeste, realizou-se 23.515.749, 26.179.779, 23.802.907, 5.915.355, 6.416.875 e 3.595.435 AA entre 2015 e 2020, respectivamente, somando 89.449.541. Conclusão: Nota-se declínio na quantidade de avaliações durante esse período nas esferas regional e estadual. No Nordeste, observou-se queda de aproximadamente $84,7 \%$ ao comparar 2015 com 2020. No Piauí, houve redução de aproximadamente 98,5\% quando comparados os $\operatorname{anos} 2015$ e 2020 . No município, observou-se queda de 6,6\%, seguida de aumento de $33,7 \%$ nos três primeiros anos. A ausência dos dados mais recentes de Parnaíba dificultou a análise a nível municipal.

Descritores: Antropometria; Epidemiologia; Atenção à Saúde.

\footnotetext{
${ }^{1}$ Discente da Universidade Federal do Delta do Parnaíba (UFDPar), Parnaíba-PI, brenovrcs@gmail.com, https://orcid.org/0000-0001-6286-9607.

${ }^{2}$ Discente da Universidade Federal do Delta do Parnaíba (UFDPar), Parnaíba-PI, isabellapires@ufpi.edu.br, https://orcid.org/0000-0003-3486-7908.

${ }^{3}$ Discente da Universidade Federal do Delta do Parnaíba (UFDPar), Parnaíba-PI, nogueirarego@ufpi.edu.br, https://orcid.org/0000-0001-6633-1425.

${ }^{4}$ Discente da Universidade Federal do Delta do Parnaíba (UFDPar), Parnaíba-PI, priscyllafrazao1995@gmail.com, https://orcid.org/0000-0001-7580-516X.

${ }^{5}$ Docente da Universidade Federal do Delta do Parnaíba (UFDPar), Parnaíba-PI, ericasilva.ma@ gmail.com, https://orcid.org/0000-0001-5003-037X.
} 


\title{
ASSISTÊNCIA DE ENFERMAGEM FRENTE AO PACIENTE POLITRAUMATIZADO EM EMERGÊNCIA HOSPITALAR: UMA REVISÃO DA LITERATURA
}

\author{
Francisco Lucas Leandro de Sousa ${ }^{1}$, Danilo da Silva Alves ${ }^{2}$
}

Introdução: O politraumatismo é caracterizado por múltiplas lesões em um episódio, desencadeado por uma troca de energia entre os tecidos e o meio. Os traumas representam um agravante para o Sistema Único de Saúde, provocando impacto significativo nas taxas de morbimortalidade e hospitalização. Objetivo: Identificar a assistência de enfermagem ao paciente politraumatizado no ambiente hospitalar de emergência. Métodos: Trata-se de uma revisão integrativa realizada a partir das bases de dados da LILACS e SCIELO através dos descritores: "Enfermagem", "Serviço Hospitalar de Emergência" e "Traumatismo Múltiplo". Como critérios de inclusão artigos originais que abordassem a temática, disponíveis online, na íntegra, nos idiomas português e inglês, entre 2017 e 2021 e como critérios de exclusão: estudos repetidos nas bases de dados. Totalizando 10 estudos para compor a revisão. Resultados: A administração do tempo na emergência é essencial para uma qualidade no atendimento, desse modo o profissional deverá seguir critérios para obter informações sistematizadas e organizadas para determinação do estado de saúde da vítima. As intervenções de enfermagem realizadas ao paciente com traumatismo múltiplo incluem: avaliação primária da vítima, administração de medicação, controle e monitorização, exames laboratoriais, cuidados com higiene, drenos, mobilização, posicionamento, vias áreas artificiais e suporte ventilatório. Nesse sentido, o profissional enfermeiro desempenha papel importante na assistência à vítima de trauma, uma vez que visa minimizar o risco de o paciente desenvolver sequelas. Conclusão: Nota-se a importância do enfermeiro no setor da emergência, com o intuito de promover assistência integral e humanizada ao paciente vítima de trauma ofertando conduta assistencial eficaz.

Descritores: Enfermagem; Serviço hospitalar de emergência; Traumatismo múltiplo.

\footnotetext{
${ }^{1}$ Centro Universitário Maurício de Nassau (UNINASSAU), Fortaleza, Ceará, Brasil. E-MAIL: lucasleandro2912@gmail.com. ORCID: https://orcid.org/0000-0003-2802-2378

${ }^{2}$ Centro Universitário Maurício de Nassau (UNINASSAU), Fortaleza, Ceará, Brasil. E-MAIL: enferdanilo.alves@gmail.com. ORCID: https://orcid.org/000-0001-7663-223X
} 


\section{CRISE ANAFILÁTICA: MANEJO NA EMERGÊNCIA MÉDICA}

Maria Hélida Alves Callou de Sá1; Bruna de Lima Pachêco ${ }^{2}$; Josevaldo Araújo de Melo ${ }^{3}$.

Introdução: A anafilaxia é uma das mais dramáticas condições clínicas da emergência médica, tanto pela imprevisibilidade do aparecimento quanto pelo potencial de gravidade da sua evolução. A conduta dos profissionais de saúde frente a esse quadro representa ponto crucial para a estabilização do paciente, com necessidade de rápido reconhecimento e manejo apropriado desta síndrome. Objetivo: Realizar uma revisão de literatura com as principais orientações de manejo do paciente com reação anafilática. Métodos: O trabalho baseou-se em artigos encontrados nas bases de dados SciELO e Google Acadêmico, utilizando os seguintes descritores "Emergências", "Terapêutica" e "Anafilaxia". Selecionou-se 3 artigos conforme critério de abordagem pertinente ao tema. Resultados: A despeito do manejo na emergência médica em pacientes que desenvolvem crise anafilática, a base para o sucesso no tratamento é a rapidez das ações. A imediata intervenção para o acesso das vias aéreas e à circulação, com o objetivo de manutenção adequada dos sinais vitais, é o primeiro passo na conduta. A adrenalina é a pedra angular no tratamento da anafilaxia, e o seu uso deve ser o mais rápido possível, levando em consideração que a evolução da crise anafilática se sucede com comprometimento respiratório e/ou cardiovascular que pode culminar com choque refratário e parada cardiorrespiratória. Intervenções subsequentes, dependendo da resposta terapêutica à adrenalina, podem incluir anti-histamínicos, corticosteróides, oxigenioterapia, expansores de volume e agentes vasopressores. Conclusão: De uma forma geral, o manejo do paciente anafilático requer manter as vias aéreas pérvias, avaliar os sinais vitais e administração imediata da adrenalina como ponto crucial na terapêutica.

Descritores: Emergências; Terapêutica; Anafilaxia.

\footnotetext{
1 Aluna do Curso de Bacharelado em Medicina da Universidade de Pernambuco; Garanhuns, PE; maria991651697@gmail.com

2 Aluna do Curso de Bacharelado em Medicina da Universidade de Pernambuco; Garanhuns, PE; resumosmedupe@gmail.com

3 Professor do Curso de Bacharelado em Medicina da Universidade de Pernambuco; Garanhuns, PE; josevaldoamelo@yahoo.com.br
} 


\section{DESAFIOS DAS PESSOAS QUE VIVEM COM HIV/AIDS FRENTE À PANDEMIA DE COVID-19: UMA REVISÃO INTEGRATIVA}

Tiago Lima Nogueira ${ }^{1}$; William Cataldo Teixeira ${ }^{2}$; Nayron Vitor do Nascimento Barbosa ${ }^{3}$, Ana Karolina Nascimento Paula ${ }^{4}$, Karina Rodrigues dos Santos ${ }^{5}$.

Introdução: A pandemia de HIV/AIDS acometeu, na década de 80, uma grande parcela da população e ainda hoje modifica as relações e a vida de inúmeras pessoas. Em 2020, com o surgimento da pandemia de Covid-19, essa população, fisiologicamente imunossuprimida, viu surgir novos desafios e barreiras a serem enfrentados nesse ambiente hostil, principalmente para essa parcela da sociedade, caracterizada pela Organização Mundial da Saúde pertencente ao grupo de risco para a nova linhagem do coronavírus. Objetivo: Analisar os desafios encontrados por pessoas que convivem com HIV/AIDS frente à pandemia do Covid-19. Método: Trata-se de uma revisão integrativa da literatura baseada na busca sistemática nas bases Lilacs e SciELO. Resultados: Diante do cenário pandêmico algumas mudanças na rotina dessa população foram evidenciadas, como o convívio familiar acentuado, devido à necessidade de isolamento social, a diminuição do acompanhamento médico, em função da situação da saúde pública e o medo gerado pela doença, visto que essas pessoas pertencem ao grupo de risco. Conclusão: Mudanças e desafios psicossociais foram vivenciados por essa parcela da população no decorrer desses últimos 2 anos, alertando, assim, para a necessidade de maior visibilidade e atenção a esse público, o qual já vem enfrenta problemas sociais desde a década de 80. Dessa forma, torna-se importante o desenvolvimento de estratégias e soluções para lidarem com a saúde dessa população durante esse momento adverso, melhorando a sua qualidade de vida e mitigando os desafios causados pela pandemia do Covid-19.

Descritores: Revisão; COVID-19; HIV.

\footnotetext{
${ }^{1}$ Universidade Federal do Delta do Parnaíba (UFDPar). Parnaíba (PI), Brasil. E-mail: tiagolima@ufpi.edu.br

${ }^{2}$ Universidade Federal do Delta do Parnaíba (UFDPar). Parnaíba (PI), Brasil. E-mail: williamcataldo1998@gmail.com

${ }^{3}$ Universidade Federal do Delta do Parnaíba (UFDPar). Parnaíba (PI), Brasil. E-mail: nayron.n.b@ufpi.edu.br

${ }^{4}$ Universidade Federal do Delta do Parnaíba (UFDPar). Parnaíba (PI), Brasil. E-mail: karolinna74@ gmail.com

${ }^{5}$ Universidade Federal do Delta do Parnaíba (UFDPar). Parnaíba (PI), Brasil. E-mail:

krsantos2004@yahoo.com.br
} 


\title{
ESTUDO COMPARATIVO DO PERFIL DE MORTALIDADE POR DOENÇAS CARDIOVASCULARES ENTRE A POPULAÇÃO DO BRASIL E DO PIAUÚ
}

\author{
Camila de Maria Ferreira Brandão ${ }^{1}$; Adrielly Cristhine Gonçalves Araujo ${ }^{2}$; Andressa \\ Carvalho Pereira $^{3}$; Olívio Joaquim Fonseca Neto ${ }^{4}$; Érica de Araújo Silva Mendes ${ }^{5}$
}

Introdução: As doenças cardiovasculares representam a principal causa de óbito no Brasil. As taxas de mortalidade por esse agravo são heterogêneas entre as microrregiões brasileiras, portanto, conhecer as particularidades locais é essencial para que medidas de prevenção e controle sejam efetivadas. Objetivo: Analisar, comparativamente, o perfil de mortalidade por doenças cardiovasculares entre residentes no Brasil e no Piauí de 2008 a 2021. Métodos: É um estudo retrospectivo de abordagem quantitativa através de dados coletados na plataforma DATASUS entre o período de janeiro de 2008 a junho de 2021. Resultados: A taxa de mortalidade por doenças cardiovasculares no Brasil foi de 8,02 por cem mil habitantes, sendo mais elevada em pessoas pretas com 8,24 e menor em brancas com 7,42. No Piauí, a taxa geral foi de 6,35, a população indígena possuindo a maior taxa, de 5,13 e pessoas brancas dispondo das menores de 3,25. A prevalência foi maior em homens com taxa de 8,15 no Brasil e 6,8 no Piauí. Quanto à idade, no Brasil, a maior taxa foi 16, em pessoas com mais de 80 anos, contudo, no Piauí, a maior taxa foi em menores de 1 ano, com 12,34. Conclusão: Houve divergência quanto à raça e faixa etária. A taxa de mortalidade por doenças cardiovasculares foi maior entre homens pretos, no Brasil, e entre homens indígenas, no Piauí. Menores de 1 ano apresentaram a maior taxa no Estado, em contraste ao padrão nacional de maior mortalidade entre idosos. Mais estudos são necessários para investigar a causa dessas disparidades.

Descritores: Doenças Cardiovasculares; Mortalidade; Estudo Comparativo.

\footnotetext{
${ }^{1}$ Camila de Maria Ferreira Brandão / Acadêmica de medicina UFDPar / Parnaíba - PI / camilamfb@ufpi.edu.br

2 Adrielly Cristhine Gonçalves Araujo/ Acadêmica de medicina UFDPar / Parnaíba - PI / adriellygoncalves@ufpi.edu.br / https://orcid.org/0000-0002-2925-2231

${ }^{3}$ Andressa Carvalho Pereira / Acadêmica de medicina - UFDPar / Parnaíba - PI / andressa_carvalho@ufpi.edu.br / https://orcid.org/0000-0001-7721-0661

${ }^{4}$ Olívio Joaquim Fonseca Neto / Acadêmico de medicina UFDPar / Parnaíba - PI / oliviofonsecaneto@ufpi.edu.br / https://orcid.org/0000-0002-2695-0077

${ }^{5}$ Érica de Araújo Silva Mendes / Médica professora UFDPar / Parnaíba - PI / ericasilva.ma@gmail.com / https://orcid.org/0000-0001-5003-037X
} 


\title{
ANÁLISE EPIDEMIOLÓGICA DAS INTERNAÇÕES DE URGÊNCIA POR FRATURA DO FÊMUR NO PIAUÍ NO PERÍODO DE 2011 A 2020
}

\author{
Bianca Lopes Cacau ${ }^{1}$, Mikaelly Melgaço Nunes ${ }^{2}$, Ivy Louise Carvalho Barbosa Barros ${ }^{3}$, \\ Carlos Antônio Soares de Sousa Filho ${ }^{4}$, Deodato Narciso de Oliveira Castro Neto ${ }^{5}$
}

Introdução: As fraturas do fêmur representam importante problema de saúde pública, interferindo significativamente na qualidade de vida, visto que ocasiona dor intensa no quadril e dificuldade de deambulação. Tal cenário pode ocorrer, principalmente, devido a quedas da própria altura em indivíduos com osteoporose. Para reduzir o risco de complicações secundárias à fratura, o tratamento deve ser realizado em até 48 horas. Objetivo: Analisar a epidemiologia das internações de urgência por fratura femoral no Piauí de 2011 a 2020. Métodos: Trata-se de uma análise quantitativa, epidemiológica, observacional e transversal dos casos de internações de urgência por fratura femoral de 2011 a 2020. Os dados foram retirados da base DATASUS, através do Sistema de Informações Hospitalares do SUS com as variáveis: sexo, faixa etária, raça/cor e óbitos. Resultados: Verificou-se o registro de 13.370 casos. Dentre os anos analisados, 2017 apresentou a maior prevalência (12,02\%), seguido de 2018 (11,68\%). Quanto ao sexo, observou-se uma maior prevalência entre o sexo masculino $(55,10 \%)$. A respeito da faixa etária, houve prevalência entre maiores de 80 anos $(23,52 \%)$, seguido por 70 a 79 anos $(14,80 \%)$. Em relação a cor/raça, observou-se que 69,252\% não foram notificados quanto a essa variável e 26,90\% relacionava-se a pardos. Foram registrados 323 óbitos, correspondendo a $2,41 \%$ dos casos. Conclusão: Dessa forma, infere-se que os homens idosos são os mais afetados. Ressalta-se a necessidade de prevenção e tratamento adequado, principalmente, neste grupo mais vulnerável.

Descritores: Ortopedia; Epidemiologia; Fratura; Fêmur.

\footnotetext{
${ }^{1}$ Universidade Federal do Delta do Parnaíba (UFDPar), Parnaíba, Piauí. E-mail: bianca.lopescacau1@ gmail.com

${ }^{2}$ Universidade Federal do Delta do Parnaíba (UFDPar), Parnaíba, Piauí. E-mail: mikaellymel123@gmail.com

${ }^{3}$ Universidade Federal do Delta do Parnaíba (UFDPar), Parnaíba, Piauí. E-mail: ivybarros14@ @otmail.com

${ }^{4}$ Universidade Federal do Delta do Parnaíba (UFDPar), Parnaíba, Piauí. E-mail: carlos17041@gmail.com

${ }^{5}$ Universidade Federal do Delta do Parnaíba (UFDPar), Parnaíba, Piauí. E-mail: deodatonarciso@hotmail.com
} 


\title{
ANÁLISE EPIDEMIOLÓGICA DOS CASOS DE HANSENÍASE NO PIAUÍ NOS ANOS DE 2015 A 2019
}

\author{
Carlos Antônio Soares de Sousa Filho ${ }^{1}$, Ingrid Brandão Cardoso Paz ${ }^{2}$, Arícia Gomes \\ Miranda ${ }^{3}$, Jamilly Santiago Rocha ${ }^{4}$, Ivan Rodrigues Silva ${ }^{5}$
}

Introdução: A hanseníase é uma doença crônica infectocontagiosa que persiste sendo um problema de saúde pública no Brasil, com uma média de 30.000 novos casos por ano. O patógeno é o Mycobacterium leprae, bactéria que atinge principalmente os olhos, nervos periféricos e pele. Sua disseminação ocorre por meio do contato próximo e contínuo com doentes não tratados. Além disso, fatores de risco socioeconômicos e pessoais podem levar à maior suscetibilidade à doença. Objetivos: Realizar delineamento epidemiológico dos casos de hanseníase notificados no Piauí nos anos de 2015 a 2019. Métodos: Estudo epidemiológico, quantitativo, observacional e transversal sobre casos de hanseníase no Piauí entre 2015 e 2019. Os dados foram coletados no Sistema Nacional de Informação sobre Doenças e Agravos de Notificação, do banco de dados do DATASUS, analisando-se as variáveis sexo e escolaridade. Resultados: Durante o período analisado houve 6.356 casos de hanseníase, 55,8\% (N=3.687) em homens e 44,2\% (N=2.669) em mulheres. O ano com maior prevalência foi 2018, com 21,2\% (N=1.347). Quanto à escolaridade, 23,4\% ( $\mathrm{N}=1.487)$ tinham, pelo menos, o ensino fundamental completo, enquanto 41,2\% (N=2.618) não tinham e 13,2\% ( $\mathrm{N}=838)$ eram analfabetos. Conclusão: Dessa forma, conclui-se que os homens com baixa escolaridade são os mais afetados. Evidencia-se portanto, a importância do diagnóstico precoce, da prevenção e do tratamento adequado, especialmente para esta parcela social mais vulnerável.

DESCRITORES: Hanseníase; Escolaridade; Epidemiologia.

\footnotetext{
${ }^{1}$ Universidade Federal do Delta do Parnaíba (UFDPar), Parnaíba, Piauí. E-mail: carlos17041@gmail.com

${ }^{2}$ Universidade Federal do Delta do Parnaíba (UFDPar), Parnaíba, Piauí. E-mail: ingridbrandaoc@gmail.com

${ }^{3}$ Universidade Federal do Delta do Parnaíba (UFDPar), Parnaíba, Piauí. E-mail: ariciamiranda05@ gmail.com

${ }^{4}$ Universidade Federal do Delta do Parnaíba (UFDPar), Parnaíba, Piauí. E-mail: santjamilly@gmail.com

${ }^{5}$ Universidade Federal do Delta do Parnaíba (UFDPar), Parnaíba, Piauí. E-mail: ivandoctor@gmail.com
} 


\section{ANÁLISE EPIDEMIOLÓGICA DOS CASOS DE INFARTO AGUDO DO MIOCÁRDIO NO PIAUÍ: UM RECORTE DO PERÍODO DE 2015 A 2020}

Ivy Louise Carvalho Barbosa Barros ${ }^{1}$, Jamilly Santiago Rocha ${ }^{2}$; Luana Mazza Malta ${ }^{3}$; Bianca Lopes $\mathrm{Cacau}^{4}$; Antônio Tiago da Silva Souza 5 .

Introdução: Infarto Agudo do Miocárdio, ou ataque cardíaco, é o processo de morte do tecido de parte do músculo cardíaco por falta de oxigênio, devido à obstrução da artéria coronária. Essa obstrução ocorre, geralmente, pela formação de um coágulo sobre uma área já comprometida por aterosclerose (placa de gordura), causando estreitamentos dos vasos sanguíneos, podendo levar à morte súbita. Objetivo: Realizar delineamento epidemiológico dos casos de infarto agudo do miocárdio no Piauí de 2015 a 2020. Métodos: Estudo epidemiológico, quantitativo, observacional e transversal, pela lista de morbidade CID-10, sobre pacientes que sofreram infarto agudo do miocárdio no Piauí entre 2015 e 2020. Os dados foram coletados no Departamento de Informática do Sistema Único de Saúde do Brasil (DATASUS), analisando as seguintes variáveis: sexo, faixa etária, caráter, cor/raça e regime. Resultados: Contabilizou-se 10.222 casos no período analisado. Desse total, 6.535 (63,93\%) representam o sexo masculino, sendo o mais prevalente, e $4.913(48,06 \%)$ estão como sem informação na classificação “cor e raça”. Vale destacar que 3.101 casos (30,34\%) estão na faixa etária de 60 a 69 anos, sendo Urgência o caráter mais preponderante, resultando em $9.579(93,71 \%)$ infartos. Quanto ao regime do atendimento, o Ignorado é o mais evidente com $9.048(88,51 \%)$. Conclusão: Conclui-se que a preponderância dos casos de ataque cardíaco ocorre em indivíduos do sexo masculino e na faixa etária entre 60 a 69 anos. Ademais, constatou-se que essa doença é uma emergência médica, especialmente devido ao seu caráter súbito e rápido, necessitando de atendimento imediato e de maior atenção.

Descritores: Infarto do Miocárdio; Epidemiologia; Sistema de Informação Hospitalar.

\footnotetext{
${ }^{1}$ Discente da Universidade Federal do Delta do Parnaíba, Parnaíba-PI, ivybarros14@ufpi.edu.br;

${ }^{2}$ Discente da Universidade Federal do Delta do Parnaíba, Parnaíba-PI, santjamilly@ gmail.com;

${ }^{3}$ Discente da Universidade Federal do Delta do Parnaíba, Parnaíba-PI, mazzamluana@gmail.com;

${ }^{4}$ Discente da Universidade Federal do Delta do Parnaíba, Parnaíba-PI, bianca.lopescacau1@ @mail.com;

${ }^{5}$ Mestre em Enfermagem pela Universidade Federal do Piauí, Teresina-PI, at.tiago@ hotmail.com.
} 


\title{
ANÁLISE EPIDEMIOLÓGICA DAS INTERNAÇÕES E DOS ÓBITOS POR INSUFICIÊNCIA RENAL NO BRASIL ENTRE OS ANOS DE 2010 E 2020.
}

\author{
Rômulo Sasso Dagostini ${ }^{1}$, Isabella Cabral Ferraz ${ }^{2}$, Felipe Henzo Carvalho Cerqueira ${ }^{3}$, Ivy \\ Louise Carvalho Barbosa Barros ${ }^{4}$, Antônio Tiago da Silva Souza ${ }^{5}$
}

Introdução: A Insuficiência Renal (IR) é um distúrbio no qual os rins perdem a capacidade de filtrar o sangue com eficiência, e de eliminar produtos de degradação metabólica prejudicando o equilíbrio de eletrólitos do corpo. A IR pode ser classificada em aguda ou crônica, e por ser uma disfunção silenciosa, quando descoberta, encontra-se em um estágio mais avançado. Objetivo: Descrever o perfil epidemiológico das internações e dos óbitos por IR no Brasil no período de 2010 a 2020. Métodos: Estudo epidemiológico, descritivo, quantitativo, e transversal decorrentes da IR no Brasil no período de 2010 a 2020. Caracterizou-se as internações e óbitos por sexo, faixa etária, raça/cor, e estado por meio de estatística e prevalência. Os dados foram obtidos no Departamento de Informática do Sistema Único de Saúde (DATASUS). Resultados: Houve um total de 1.118.070 casos de internações por IR no Brasil, equivalente a uma média de 101.642 casos/anos. O estado com maior prevalência de internações foi São Paulo com 20\% (n=2019.649). Entre total de internações, observou-se um predomínio do sexo masculino 57\% (n=633.498) e da faixa etária entre 60 e 69 anos 22\% $(n=242.354)$. Do total de internações 13\% resultaram em óbitos $(n=139.979)$. Segundo a variável raça/cor, identificou-se 36\% para a população branca $(n=405.962)$. O ano preponderante foi 2019 com 11\% (n=121.671). Conclusão: O aumento do número de internações e óbitos entre idosos pode ser consequência do diagnóstico tardio da IR que tem um agravamento exponencial de acordo com o tempo em que não é tratada.

Descritores: Epidemiologia; Sistemas de Informação Hospitalar; Insuficiência Renal.

\footnotetext{
${ }^{1}$ Discente da Universidade Federal do Delta do Parnaíba, Parnaíba/PI, romulosdagostini@gmail.com

${ }^{2}$ Discente da Universidade Federal do Delta do Parnaíba, Parnaíba/PI, isabellacferraz17@gmail.com

${ }^{3}$ Discente da Universidade Federal do Delta do Parnaíba, Parnaíba/PI, felipehenzo@ufpi.edu.br

${ }^{4}$ Discente da Universidade Federal do Delta do Parnaíba, Parnaíba/PI, ivybarros14@hotmail.com

${ }^{5}$ Mestre em Enfermagem pela Universidade Federal do Piauí, Teresina-PI, Discente do curso de Medicina da UFDPar, Parnaíba-PI, at.tiago@hotmail.com
} 


\title{
ANÁLISE EPIDEMIOLÓGICA COMPARATIVA ENTRE O NÚMERO DE INTERNAÇÕES E ÓBITOS POR NEOPLASIA MALIGNA DA MAMA, NA REGIÃO DA PLANÍCIE LITORÂNEA, NO PERÍODO DE 2015 À 2020
}

\author{
Felipe Henzo Carvalho Cerqueira ${ }^{1}$, Romulo Sasso D’Agostini ${ }^{2}$, Lucas Marques Santiago ${ }^{3}$, \\ Luiz Henrique Sousa Oliveira ${ }^{4}$, Ivan Rodrigues Silva ${ }^{5}$.
}

Introdução: Neoplasia maligna da mama é caracterizada pela proliferação descontrolada de células nessa região, com o passar do tempo, a multiplicação das células cancerígenas pode espalhar-se para outros órgãos e tecidos, caracterizando metástase, que acaba por dificultar o tratamento e aumentar a possibilidade de óbito. Objetivos: Correlacionar a incidência anual de internações por neoplasia maligna da mama, com sua taxa de óbito, no período de 2015 a 2020. Métodos: Trata-se de um estudo epidemiológico e descritivo cujos dados foram obtidos por meio de consulta a dados do Departamento de Informática do Sistema Único de Saúde (DATASUS), acessado em abril de 2021. A população do estudo constituiu-se por internados devido a neoplasia maligna da mama, em conjunto de pessoas que vieram a óbito por essa neoplasia, na Região de Saúde da Planície Litorânea, estado do Piauí, no período de 2015 à 2020. Resultados: De acordo com o DATASUS, nesse período, o número de internações teve um aumento de 4100\%, salto de 1 caso em 2015, para 42 em 2020. Em comparação, a taxa de óbito foi de 1 caso em 2015, para 2 em 2020, configurando um aumento de 100\%. Conclusão: Os dados permitem inferir que, o crescimento exacerbado do número de casos de internação, não se refletiu em uma elevação proporcional na taxa de óbito, devido possivelmente, a uma maior realização de exames precoces de mama, uma vez que, quanto mais cedo se diagnostica neoplasias, maior a chance de sobrevida.

Descritores: Epidemiologia; Neoplasias da mama; Sistemas de Informação Hospitalar.

\footnotetext{
${ }^{1}$ Felipe Henzo Carvalho Cerqueira; Universidade Federal do Delta do Parnaíba; Parnaíba-PI; felipehenzo@ufpi.edu

${ }^{2}$ Romulo Sasso D’Agostini; Universidade Federal do Delta do Parnaíba; Parnaíba-PI; romulosdagostini@gmail.com

${ }^{3}$ Lucas Marques Santiago; Universidade Federal do Delta do Parnaíba; Parnaíba-PI; lucasmsantiago@hotmail.com

${ }^{4}$ Luiz Henrique Souza Oliveira; Universidade Federal do Delta do Parnaíba; Parnaíba-PI; luiz.hsoliveira00@gmail.com

${ }^{5}$ Ivan Rodriguez Silva; Universidade Federal do Delta do Parnaíba; Parnaíba-PI; ivandoctor@gmail.com
} 


\title{
INTUBAÇÃO SUBMENTONIANA PARA MANEJO DAS VIAS AÉREAS EM PACIENTES COM FRATURAS COMPLEXAS DE FACE.
}

\author{
Maria Alice da Silva Souza ${ }^{1}$; Carolina Pereira da Silva ${ }^{2}$; Shamara Pinto Ferreira da Cruz ${ }^{3}$; \\ Julia Soledade Vieira de Mello ${ }^{4}$; Isaque Ferreira Soares ${ }^{5}$.
}

Introdução: As fraturas complexas da face acometem a porção óssea dos terços superior, médio e inferior, e tornam o manejo da via aérea um grande desafio. Muitas vezes, a intubação faz-se necessária e suas técnicas são variadas de acordo com as necessidades do paciente. Em pacientes com trauma de face, quando as intubações oro e nasotraqueais são contraindicadas, pode-se realizar a traqueostomia. Porém, esse método acarreta uma maior morbilidade, tornando a técnica submentoniana uma melhor alternativa. Objetivo: Este estudo visa realizar uma revisão bibliográfica sobre a intubação submentoniana para o manejo das vias aéreas em pacientes com fraturas complexas de face. Métodos: Realizou-se uma revisão literária através do cruzamento dos descritores selecionados em busca eletrônica entre os anos de 2016 e 2021, encontrados nos periódicos das bases de dados SCIELO, LILACS, SCOPUS e MEDLINE. Resultados: A intubação submentoniana por ser um procedimento simples, rápido e de baixa morbidade, é relatada como uma alternativa à traqueostomia, pois apresenta menores complicações. Essa intubação promove uma via aérea mais segura e permeável, permitindo manipulação da oclusão dentária e o acesso às fraturas naso-órbito-etmoidais. Também evita os riscos potenciais de meningite iatrogênica ou trauma à região anterior da base do crânio. A técnica consiste na realização da intubação orotraqueal seguida de um acesso em região submentoniana extra-oral. Não apresenta complicações importantes relatadas, além de menor tempo e menor curso. Conclusão: A intubação submentoniana é uma técnica rápida, simples e com poucas complicações, considerada segura e eficaz em pacientes com fraturas complexas na face.

Descritores: Emergências; Intubação; Traumatismos Faciais.

\footnotetext{
${ }^{1}$ Graduanda em Odontologia pela Universidade de Pernambuco, Recife, PE. E-mail: alice.ssouza@upe.br

2 Graduanda em Odontologia pela Universidade de Pernambuco, Recife, PE. E-mail: carolinapereeira@gmail.com

${ }^{3}$ Graduanda em Odontologia pela Universidade de Pernambuco, Recife, PE. E-mail:shamara.cruz@upe.br

${ }^{4}$ Graduanda em Odontologia pela Universidade de Pernambuco, Recife, PE. E-mail: julia.soledade@upe.br

5 Docente do Centro Universitário Facol (UNIFACOL), Vitória de Santo Antão, PE. E-mail: Isaqueodontolab@hotmail.com
} 


\section{MANEJO E ACESSO ÀS VIAS AÉREAS DE PACIENTES COM TRAUMA DE FACE EM SITUAÇÕES DE EMERGÊNCIA}

Lohana Maylane Aquino Correia de Lima ${ }^{1}$, Ricardo Eugenio Varela Ayres de Melo².

Introdução: O trauma de face, necessita de uma excessiva atenção de quem atende, uma vez que a via aérea humana é um sistema composto de importantes estruturas. O acesso das vias aéreas tem prioridade em quase todos os pacientes graves segundo o protocolo do ATLS. Objetivo: Apresentar as manobras de acesso às vias aéreas nas emergências de pacientes com trauma de face. Métodos: Realizou-se uma revisão de literatura no SciELO e no PubMed. Utilizou-se os descritores "Airway Obstruction" e "Emergency" entre 2015 a 2020 com 45 artigos triados. Resultados: A manobra de Jaw Thrust é caracterizada pela protrusão da mandíbula e a de Chin Lift é a hiperextensão cervical com elevação do mento. A Intubação Endotraqueal garante o fluxo do ar por meio da aposição de uma cânula oral ou nasal. Nos procedimentos invasivos, a cricotireoidostomia cria uma abertura através da membrana cricotireoidea enquanto que a traqueostomia consiste na criação de uma abertura na traqueia, realizada entre o $2^{\circ}$ e o $3^{\circ}$ anel traqueal. Por isso, as manobras de Jaw Thrust, Chin Lift e a intubação endotraqueal são as primeiras eleitas para abertura das vias aéreas. Nos procedimentos invasivos, a cricotireoidostomia é a primeira escolha para as situações de emergência, seguida da Traqueostomia, de caráter eletivo, pois demanda maior tempo de procedimento, infraestrutura e experiência profissional. Conclusão: $\mathrm{O}$ adequado manejo das vias aéreas nas emergências é imprescindível por parte do Cirurgião Buco Maxilo Facial, pois a identificação precoce do comprometimento das vias aéreas reduzirá o potencial de danos nessas situações.

Descritores: Manuseio das Vias Aéreas; Face; Traumatologia.

\footnotetext{
${ }^{1}$ Universidade Federal de Pernambuco (UFPE) - Recife, Pernambuco, Brasil - Lohanawatson@ hotmail.com ORCID: 0000-0002-1864-8329

${ }^{2}$ Universidade Federal de Pernambuco (UFPE) - Recife, Pernambuco, Brasil - revamelo@yahoo.com ORCID: 0000-0003-1401-457X
} 


\section{MANIFESTAÇÕES CLÍNICAS DA COVID-19 EM GESTANTES: UMA REVISÃO SISTEMÁTICA.}

Simone Santos e Silva Melo ${ }^{1}$, Sarah Nilkece Mesquita Araújo Nogueira Bastos ${ }^{2}$, Bárbara Louise Freire Barbosa ${ }^{3}$, Larisse Giselle Barbosa Cruz ${ }^{4}$, Caroline Camargo Bandeira da Silveira $\mathrm{Luz}^{5}$

Introdução: A pandemia COVID-19 afeta, especialmente, pacientes em grupos de alto risco, dentre eles, as gestantes. Objetivo: Analisar os sinais e sintomas apresentados por gestantes com COVID-19. Método: Trata-se de uma revisão sistemática da literatura nas bases de dados: MEDLINE/PubMed, LILACS, SCIELO e CNKI. Os descritores controlados, utilizados na estratégia de busca nos idiomas inglês e português foram: "Gravidez/Pregnancy”; "2019nCov";"SARS-Cov-2"; Coronavírus/Coronavirus". Elegeram-se títulos originais, sem restrição de idioma e período, e que abordassem gestantes com diagnóstico clínico e/ou laboratorial de COVID-19. Excluíram-se revisões, editoriais e títulos duplicados. Resultados: Foram incluídos 34 artigos, com 412 gestantes infectadas pelo SARS-Cov-2, com idade média de 27,5 anos e média de 36,0 semanas gestacionais. Os estudos mostraram que os sinais e sintomas mais incidentes, em gestante com COVID-19 foram: febre (49,7\%;205), dispneia $(31,5 \% ; 130)$, tosse $(26,5 \% ; 109)$, fadiga $(8,2 \% ; 34)$, mialgia $(7,0 \% ; 29)$, dor torácica $(5,5 \% ; 23)$, diarreia $(4,8 \% ; 20)$ e odinofagia $(3,6 \% ; 15)$. O sintoma mais incidente foi a febre, e 21,6\%(89) de gestantes evoluíram para pneumonia viral grave. Conclusão: Gestantes com COVID-19 apresentaram manifestações clínicas semelhantes, comparadas com pacientes não grávidas. Entretanto mulheres grávidas devem receber uma atenção maior, devido às respostas imunes e a elevada patogenicidade do vírus.

Descritores: Gravidez/Pregnancy; 2019-nCov; SARS-Cov-2; Coronavírus/Coronavirus.

\footnotetext{
${ }^{1}$ Graduanda de Medicina da Universidade Federal do Delta do Parnaíba, Parnaíba (PI); simonesantosesilva@yahoo.com.br;

${ }^{2}$ Graduanda de Medicina da Universidade Federal do Delta do Parnaíba, Parnaíba (PI); sarahnilkece@hotmail.com;

${ }^{3}$ Graduanda de Medicina da Universidade Federal do Delta do Parnaíba, Parnaíba (PI); barbaralouise80@gmail.com;

${ }^{4}$ Graduanda de Medicina da Universidade Federal do Delta do Parnaíba, Parnaíba (PI); larissegiselle@hotmail.com;

${ }^{5}$ Docente da Universidade Federal do Delta do Parnaíba, Parnaíba(PI); carolinecbsilveira@gmail.com;
} 


\title{
ANÁLISE EPIDEMIOLÓGICA DA MORTALIDADE MATERNA POR ECLÂMPSIA NO ESTADO DO PIAUÍ ENTRE OS ANOS DE 2015 E 2019
}

\author{
Martha Laura Leão dos Santos Silva ${ }^{1}$, Livia Rocha Santos ${ }^{2}$, Igor dos Santos Cavalcante ${ }^{3}$, Ana \\ Vitória Meireles Veiga ${ }^{4}$, Caroline Camargo Bandeira da Silveira Luz ${ }^{5}$
}

Introdução: A doença hipertensiva gestacional está entre as três principais causas de óbitos maternos no mundo. Muitas destas são evitáveis, sendo necessário conhecimento do manejo adequado da doença e do perfil epidemiológico. A pré-eclâmpsia se manifesta predominantemente por hipertensão e proteinúria após 20 semanas e alguns fatores de risco são obesidade e diabetes mellitus. Já a eclâmpsia, agravamento da pré-eclâmpsia, caracterizase por crises convulsivas. Objetivo: Analisar os óbitos maternos por eclâmpsia no estado do Piauí entre os anos de 2015 e 2019. Métodos: Trata-se de uma pesquisa epidemiológica na qual as informações foram extraídas da base de dados Estatísticas vitais disponibilizada pelo sistema DATASUS/e-SUS. Resultados: Dos 197 óbitos maternos encontrados neste período, 27 ocorreram devido à eclâmpsia, equivalente à 13,7\% do total. Destes, 33\% ocorreram durante a gravidez, $30 \%$ no trabalho de parto, $15 \%$ no puerpério e $22 \%$ não foram especificados quanto ao período. Em relação à idade, $4 \%$ dos óbitos ocorreram na faixa etária de 10 a 14 anos, $11 \%$ na de 15 a $19,37 \%$ de 20 a 29, $44 \%$ de 30 a 39 e $4 \%$ de 40 a 49. Conclusão: As mortes maternas por eclâmpsia ocorreram majoritariamente durante a gravidez, possivelmente por baixa assistência pré-natal. Em segundo lugar, aconteceram durante o trabalho de parto, que pode ter relação com administração incorreta do sulfato de magnésio, útil para prevenção das convulsões. Quanto à faixa etária, o maior número de óbitos se deu de 30 a 39 anos, provavelmente pela idade avançada ser fator de risco.

Descritores: Mortalidade Materna; Pré-Eclâmpsia; Hipertensão.

\footnotetext{
${ }^{1}$ Universidade Federal do Delta do Parnaíba (UFDPar), Parnaíba, Piauí. E-mail: marthalaura928@gmail.com ${ }^{2}$ Universidade Federal do Delta do Parnaíba (UFDPar), Parnaíba, Piauí. E-mail: liviarocha063@gmail.com

${ }^{3}$ Universidade Federal do Delta do Parnaíba (UFDPar), Parnaíba, Piauí. E-mail: igorsc@live.com

${ }^{4}$ Universidade Federal do Delta do Parnaíba (UFDPar), Parnaíba, Piauí. E-mail: vitmei@ @otmail.com

${ }^{5}$ Universidade Federal do Delta do Parnaíba (UFDPar), Parnaíba, Piauí. E-mail:

dracarolinesilveira@outlook.com
} 


\section{INTERNAÇÕES E TAXA DE MORTALIDADE POR NEOPLASIA MALIGNA DE PRÓSTATA NO PIAUÍ DE 2016 A 2020}

Luiz Henrique Sousa Oliveira ${ }^{1}$, Antônio Tiago da Silva Souza ${ }^{2}$, Alysson Leunam Meneses Vasconcelos $^{3}$, Felipe Henzo Carvalho Cerqueira ${ }^{4}$, Rômulo Sasso Dagostini ${ }^{5}$.

Introdução: Considerada uma doença de alta incidência, o câncer de próstata é visto como um problema de saúde pública no Brasil, esta patologia é a quinta causa de mortes relacionadas ao câncer na população masculina mundial e o segundo tumor com maior incidência em homens no Brasil. Objetivo: Descrever o perfil epidemiológico das internações por neoplasia maligna de próstata no Piauí, entre os anos 2016 e 2020, bem como sua taxa de mortalidade. Metodologia: Estudo epidemiológico, descritivo, retrospectivo, com dados do Sistema de Informação Hospitalar do SUS do Departamento de Informática do Sistema Único de Saúde do Brasil (DATASUS) e posteriormente tabulados no Microsoft Excel®. Resultados: No período, foram registradas 1.848 internações no Piauí, as quais representam 4,87\% das 37.498 internações registradas no Nordeste. Nas internações encontraram-se majoritariamente indivíduos acima dos 50 anos, especialmente na faixa de 60 a 79 anos com 1419 internações (76,8\%). Em contrapartida, a taxa de mortalidade dessa faixa foi de $4,02 \%$ de 60 a 69 anos e 4,70\% na faixa de 70 a 79 anos, enquanto a taxa da população acima dos 80 anos chegou a uma média de $10,71 \%$. Conclusão: Constatou-se preponderância masculina, com idade entre 60 a 79 anos, e evidenciou-se a elevada mortalidade na faixa mais idosa, pois a taxa de mortalidade chegou a dobrar na população acima dos 80 anos. Esse levantamento salienta o maior risco à população mais idosa, justificando maior atenção a esses cidadãos.

Descritores: Epidemiologia; Neoplasias da Próstata; Sistema de Informação Hospitalar.

\footnotetext{
${ }^{1}$ Universidade Federal do Delta do Parnaíba - Parnaíba/PI - luiz.hsoliveira00@gmail.com

${ }^{2}$ Médico pela UNITEPCI. Docente do curso de Medicina da UFDPar - Parnaíba/PI - ivandoctor@ gmail.com

${ }^{3}$ Universidade Federal do Delta do Parnaíba - Parnaíba/PI - alyssonleunam@ hotmail.com

${ }^{4}$ Universidade Federal do Delta do Parnaíba - Parnaíba/PI - andrezamaranta16@ gmail.com

${ }^{5}$ Universidade Federal do Delta do Parnaíba - Parnaíba/PI - priscyllafrazao1995@gmail.com
} 


\title{
O ADOECIMENTO E OS PROCESSOS DE LUTO NO CONTEXTO DE PANDEMIA DE COVID-19: UMA REVISÃO INTEGRATIVA
}

\author{
Nayron Vitor do Nascimento Barbosa ${ }^{1}$, William Cataldo Teixeira ${ }^{2}$, Tiago Lima Nogueira ${ }^{3}$, \\ Ana Karolina Nascimento Paula ${ }^{4}$, Karina Rodrigues dos Santos ${ }^{5}$
}

Introdução: A pandemia de COVID-19 alterou as dinâmicas e relações humanas de todo tipo em escala mundial. Devido aos protocolos de segurança adotados frente a esse cenário, famílias têm experienciado os processos de adoecimento, morte e luto de seus entes de uma forma singular, o que tem impactado fortemente a saúde mental da população. Objetivos: Analisar os processos de adoecimento e luto no contexto de pandemia de COVID-19. Métodos: Este estudo trata-se de uma revisão integrativa que se propôs a fazer uma análise a partir da literatura nacional e internacional correspondentes aos últimos três anos disponíveis nas bases de dados LILACS, Google Scholar, BVS e SCIELO. Resultados: O arcabouço teórico apontou que a impossibilidade da proximidade física uns com os outros no cenário da COVID-19, cumprindo os variados ritos de despedida daqueles em situações como a iminência da morte bem como da realização de rituais funerários, abraçando os mais próximos, em momentos comuns de sofrimento, alteraram a subjetividade e a cultura do rito funeral e do luto, proporcionando ainda mais tristeza, dor e desolação. Conclusão: Compreende-se que as pessoas que vivenciam o luto, necessitam de alternativas de suporte, que podem ser desenvolvidas por meio de espaços sociais que possibilitem a expressão de sentimentos e a troca de experiências relacionados ao processo de perda, na tentativa de processá-lo simbolicamente. Além disso, os princípios dos cuidados paliativos são essenciais para enfrentar os desafios de uma crise humanitária.

Descritores: Enlutamento; COVID-19; Saúde Mental; Cuidado Paliativo.

\footnotetext{
${ }^{1}$ Universidade Federal do Delta do Parnaíba (UFDPar). Parnaíba (PI), Brasil. E-mail: nayron.n.b@ufpi.edu.br

${ }^{2}$ Universidade Federal do Delta do Parnaíba (UFDPar). Parnaíba (PI), Brasil. E-mail: williamcataldo1998@gmail.com

${ }^{3}$ Universidade Federal do Delta do Parnaíba (UFDPar). Parnaíba (PI), Brasil. E-mail: tiagolima@ufpi.edu.br

${ }^{4}$ Universidade Federal do Delta do Parnaíba (UFDPar). Parnaíba (PI), Brasil. E-mail: karolinna74@gmail.com

${ }^{5}$ Universidade Federal do Delta do Parnaíba (UFDPar). Parnaíba (PI), Brasil. E-mail:

krsantos2004@yahoo.com.br
} 


\title{
MANIFESTAÇÕES CLÍNICAS, DIAGNÓSTICO E TRATAMENTO PARA A PARALISIA DE BELL
}

\author{
Maria Alice da Silva Souza ${ }^{1}$; Carolina Pereira da Silva ${ }^{2}$; Shamara Pinto Ferreira da Cruz ${ }^{3}$; \\ Melissa Noêmia Barbosa da Silva ${ }^{4}$; Isaque Ferreira Soares ${ }^{5}$.
}

Introdução: A Paralisia de Bell (PB) é uma alteração idiopática, periférica e unilateral da face. Essa patologia é o tipo de paralisia mais comum relacionada ao nervo facial, representando de 60 a $75 \%$ dos casos documentados. A PB acomete majoritariamente indivíduos adultos de ambos os sexos e encontra-se comumente associada a inflamação e edema na região do gânglio geniculado do nervo facial decorrente de presença de diversas classes virais, gravidez, diabetes melittus, isquemia vascular e doenças autoimunes. Objetivo: Determinar as características, diagnóstico e tratamento para a paralisia de Bell. Métodos: O estudo baseou-se em uma revisão de literatura na qual realizou-se o cruzamento de descritores nas bases de dados Lilacs e Scielo no período de 2016 a setembro de 2021. Resultados: Entre as principais características da PB está a fraqueza unilateral e súbita dos músculos da expressão facial, hiperacusia, paresia e dor retroauricular. A gravidade desta patologia é geralmente avaliada na escala de House e Brackmann, exames diagnósticos como ressonância magnética, hemograma e punção lombar podem ser solicitados. Geralmente, esse quadro se resolve sem tratamento, no entanto, manejo com aciclovir para quadros virais e corticosteroides como a prednisona são administrados para melhora do quadro inflamatório são normalmente indicados para diagnósticos realizados dentro de 72 horas do início dos sintomas. Conclusão: É fundamental o conhecimento e acompanhamento dos sinais e sintomas dessa patologia, uma vez que o diagnóstico precoce e o início imediato do tratamento com as terapias corticoides e antivirais podem contribuir para uma melhora significativa do quadro clínico.

Descritores: Paralisia de Bell; Doenças do nervo facial; Nervo facial.

\footnotetext{
${ }^{1}$ Graduanda em Odontologia pela Universidade de Pernambuco, Recife, PE. E-mail: alice.ssouza@upe.br

2 Graduanda em Odontologia pela Universidade de Pernambuco, Recife, PE. E-mail: carolinapereeira@gmail.com

${ }^{3}$ Graduanda em Odontologia pela Universidade de Pernambuco, Recife, PE. E-mail:shamara.cruz@upe.br

${ }^{4}$ Graduanda em Odontologia pela Universidade de Pernambuco, Recife, PE. E-mail: melissa.nbsilva@upe.br

5 Docente do Centro Universitário Facol (UNIFACOL), Vitória de Santo Antão, PE. E-mail: Isaqueodontolab@hotmail.com
} 


\section{PARTICULARIDADES DO TRAUMA BUCO MAXILO FACIAL QUE DIFICULTAM O ATENDIMENTO DE EMERGÊNCIA}

Thayná Lacerda Almeida ${ }^{1}$; Evellyn Maria Silva de Almeida ${ }^{2}$; Lohana Maylane Aquino Correira de Lima ${ }^{3}$, Ricardo Eugenio Varela Ayres de Melo ${ }^{4}$.

Introdução: O Trauma Buco Maxilo Facial é uma modalidade de trauma que apresenta diversos agravantes à apresentação clínica, manejo e tratamento. Este tipo de trauma afeta tanto tecidos moles quanto estruturas ósseas, sendo sua reabilitação um processo demorado e custoso. Objetivo: O objetivo deste trabalho é identificar principais agravos do trauma facial que impedem à instituição de procedimentos de suporte básico à vida. Método: Realizou-se uma revisão de literatura selecionando artigos que tratassem de atendimentos emergenciais em vítimas de trauma facial. Resultados: A vítima de trauma facial apresenta agravos na avaliação inicial, que dificultam ou impossibilitam alguns dos métodos da Advanced Trauma Life Suport (ATLS), consagrado no tratamento de traumatismo. Na avaliação primária da vítima é preconizado o uso do padrão ABCDE. Sendo o acesso e manutenção das vias aéreas com proteção da coluna cervical, ou "A", o maior obstáculo, até mesmo porque manobras como Chin Lift e Jaw Thrust são difíceis em certos tipos de trauma. Há obstáculos ainda na instituição dos procedimentos das etapas de respiração e ventilação, ou "B"; em "C" é prioritário o controle hemorrágico; "D" avalia o nível de consciência do paciente e "E" representa expor o paciente, ou seja despi-lo e controlar o ambiente prevenindo a hipotermia. Além disso, encontra-se dificuldade na criação de uma via aérea temporária. Conclusão: Conclui-se que a vítima de trauma facial apresenta fatores agravantes que devem ser conhecidos pelo socorrista e profissional responsável pelo tratamento, a fim de otimizar o quadro, a evolução do paciente e evitar danos secundários.

Descritores: Cuidados de Suporte Avançado de Vida no Trauma; Cirurgiões Bucomaxilofaciais; Trauma; Manuseio das Vias Aéreas.

\footnotetext{
${ }^{1}$ Acadêmica do curso de Odontologia da Universidade Federal de Pernambuco, Recife - PE, thayna.lacerda@ufpe.br.

${ }^{2}$ Acadêmica do curso de Odontologia da Universidade Federal de Pernambuco, Recife - PE, evellynmsa@gmail.com.

${ }^{3}$ Mestranda em Clínica Integral pela Universidade Federal de Pernambuco, Recife - PE, lohanawatson@hotmail.com.

${ }^{4}$ Coordenador do curso de Especialização em Cirurgia e Traumatologia Buco Maxilo Facial pela Universidade Federal de Pernambuco, Recife - PE, revamelo@yahoo.com.
} 


\section{ANÁLISE DA PREVALÊNCIA DE HIPERTENSÃO ARTERIAL SISTÊMICA EM CRIANÇAS E ADOLESCENTES: REVISÃO INTEGRATIVA}

Guilherme Augusto Silva de Moraes ${ }^{1}$; Sarah Nilkece Mesquita Araujo Nogueira Bastos²; Bárbara Louise Freire Barbosa ${ }^{3}$; Gabriela Alves de Araújo ${ }^{4}$; Karina Rodrigues dos Santos $^{5}$

Introdução: Hipertensão Arterial Sistêmica (HAS) é uma doença crônica de elevado impacto sobre a saúde pública mundial. Possui alta prevalência e estreita relação com patologias cardiovasculares. Objetivo: Caracterizar e analisar a prevalência de HAS em crianças e adolescentes. MÉTODOS: Realizou-se uma revisão integrativa de literatura nas bases de dados MEDLINE, Lilacs e Scielo, por meio dos seguintes descritores: hipertensão arterial, criança, adolescente, adolescência, infância. Foram selecionados 11 artigos originais publicados de 2015 a 2020 nos idiomas português, inglês e espanhol. Resultados: Dos 11 artigos selecionados, 7 foram produzidos no Brasil, 2 no Uruguai, 1 nos Estados Unidos e 1 na Itália. Todos eram descritivos e com abordagem quantitativa. Somando-se todos os estudos, a população analisada foi de 30.776 pessoas com idades entre 4 a 19 anos. Detectaram-se 1.923 (6,2\%) crianças e adolescentes hipertensos e 340 (1,1\%) pré-hipertensos. A maior prevalência de indivíduos com HAS ocorreu em um estudo realizado no Paraná em $2020(40,5 \%)$, ao passo que a menor foi observada em um estudo norte-americano do mesmo ano $(2,7 \%)$. Alguns artigos demonstraram que a prevalência geral desta condição está crescendo dentro do grupo etário analisado. Um dos estudos sugeriu influência da raça/etnia na ocorrência de hipertensão entre adolescentes. Conclusão: Observou-se uma prevalência geral relevante e crescente de HAS em crianças e adolescentes. Esta recorrência se mostrou, no entanto, desigual em diferentes localidades e grupos étnicos, tornando pertinentes novos estudos sobre o tema.

Descritores: Adolescente; Criança; Hipertensão.

\footnotetext{
${ }^{1}$ Universidade Federal do Delta do Parnaíba (UFDPar), Parnaíba, Piauí. E-mail: guilherme_moraes@outlook.com

${ }^{2}$ Universidade Federal do Delta do Parnaíba (UFDPar), Parnaíba, Piauí. E-mail: sarahnilkece@ hotmail.com

${ }^{3}$ Universidade Federal do Delta do Parnaíba (UFDPar), Parnaíba, Piauí. E-mail: barbaralouise80@ gmail.com

${ }^{4}$ Centro Universitário Maurício de Nassau (UNINASSAU), Parnaíba, Piauí. E-mail: gabrielaaraujoa@outlook.com

${ }^{5}$ Universidade Federal do Delta do Parnaíba (UFDPar), Parnaíba, Piauí. E-mail: krsantos2004@yahoo.com.br
} 


\title{
PRINCIPAIS IMPLICAÇÕES DO TRATAMENTO ODONTOLÓGICO EM PACIENTES COM SÍNDROME DE MOEBIUS
}

\author{
Rayane Pereira de Araújo ${ }^{1}$, Thayná Lacerda Almeida ${ }^{2}$, Evellyn Maria Silva de Almeida ${ }^{3}$, \\ Lohana Maylane Aquino Correia de Lima ${ }^{4}$, Ricardo Eugenio Varela Ayres de Melo 5 .
}

Introdução: A Síndrome de Moebius (SM) é uma patologia de condição neurológica congênita rara, não progressiva, de severidade variada, caracterizada pela paralisia unilateral ou bilateral dos nervos cranianos facial e abducente. As manifestações clínicas e orais se apresentam de formas diversas e podem ser observadas as seguintes: micrognatismo, microstomia, supercrescimento da maxila o que leva a uma mordida profunda, palato profundo e atrofia da língua. Objetivos: Esta revisão de literatura tem a finalidade de descrever as características da Síndrome de Moebius e suas principais implicações na odontologia. Métodos: Foi realizada uma busca de dados nas plataformas: Pubmed, Medline e ScienceDirect, nos idiomas português e inglês, sendo selecionados 8 artigos dos últimos 10 anos. Resultados: Como a Síndrome de Moebius afeta a musculatura facial pode dificultar o tratamento odontológico devido às pequenas dimensões e pouca mobilidade muscular da cavidade bucal, assim como o ressecamento da mucosa labial e da diminuição do fluxo da saliva afetados pela atrofia do nervo facial. O ambiente bucal torna-se favorável ao desenvolvimento da cárie e da doença periodontal devido a dificuldade no autocuidado por falta de coordenação motora e/ou por retardo cognitivo, e a precária higienização devido a microstomia, aliadas ao maior tempo de permanência do alimento em contato com os dentes por causa da disfagia. Conclusão: A realização de procedimentos odontológicos em pacientes portadores da síndrome de Moebius torna-se complexo devido à sua condição física e cognitiva. É importante prestar atendimento precoce a essas crianças com dificuldade de higienização e elevado risco de cárie.

Descritores: Síndrome de Moebius, Paralisia Facial, Paralisia de nervos cranianos, Tratamento Odontológico.

\footnotetext{
${ }^{1}$ Acadêmica em odontologia, Universidade Federal de Pernambuco (UFPE), Recife - PE. rayodonto111@outlook.com

${ }^{2}$ Acadêmica em odontologia, Universidade Federal de Pernambuco (UFPE), Recife - PE. thaynalacerda955@gmail.com

${ }^{3}$ Acadêmica em odontologia, Universidade Federal de Pernambuco (UFPE), Recife - PE. evellynmsa@gmail.com

${ }^{4}$ Cirurgiã-dentista, Universidade Federal de Pernambuco (UFPE), Recife - PE. 1ohanawatson@ hotmail.com

${ }^{5}$ Coordenador do curso de Especialização em Cirurgia Buco Maxilo Facial, Universidade Federal de

Pernambuco (UFPE), Recife - PE.revamelo@yahoo.com
} 


\title{
PROCEDIMENTOS E CUSTOS HOSPITALARES ACERCA DA REALIZAÇÃO DE PAROTIDECTOMIA PARCIAL OU SUBTOTAL NO ÂMBITO DO SISTEMA ÚNICO DE SAÚDE NA REGIÃO NORDESTE NO PERÍODO DE 2011 A 2019.
}

\author{
Gildemar da Silva Lustosa Junior ${ }^{1}$, Lucas Marques Santiago ${ }^{2}$, Claudia Lima Mascarenhas \\ Diniz $^{3}$, Ian Carlos de Oliveira Andrade ${ }^{4}$, Antônio Tiago da Silva Souza ${ }^{5}$
}

Introdução: A parotidectomia diz respeito ao procedimento cirúrgico de ressecção da glândula parótida. Essa cirurgia é indicada devido à presença de nódulos na parótida e pode ser do tipo parcial, superficial ou total dependendo da doença a ser tratada. Objetivo: analisar os procedimentos de parotidectomia parcial ou subtotal e do custo desses na região nordeste no período de 2011 a 2019. Métodos: Trata-se de um levantamento epidemiológico, com abordagem quantitativa, que utilizou o banco de dados do Sistema de Informações Hospitalares do Sistema Único de Saúde (SIH-SUS) avaliando-se as variáveis: número de internações, valor total das internações, tempo médio de internação e o valor médio por internação. Resultados: De 2011 a 2019 houve 1674 internações para a realização de parotidectomia na região Nordeste. Comparando com $2011 \quad(n=78)$, observou-se um acréscimo no número de internações de 201,28\% no ano de 2018 (n=235) e 191,03\% no ano de 2019 (n=227). O total de internações custou à saúde pública $\mathrm{R} \$$ 841.170,67 no período analisado. O tempo médio de internação foi de 2,2 dias e o valor médio por internação foi de R \$ 502,49. Conclusão: Nesse sentido, uma vez que há um crescimento contínuo e potencial de malignização com consequente aumento do ônus à saúde pública, é importante que esses pacientes sejam diagnosticados e tratados precocemente, visto que a parotidectomia parcial possui um valor baixo se comparado com o mesmo procedimento em pacientes oncológicos (em média $\mathrm{R} \$ 1.255,90$ a mais).

Descritores: Epidemiologia; Sistemas de Informação Hospitalar; Custos de Cuidados de Saúde.

\footnotetext{
${ }^{1}$ Discente da Universidade Federal do Delta do Parnaíba, Parnaíba/PI, gildemarlustosa@ufpi.edu.br, https://orcid.org/0000-0003-4937-214X

${ }^{2}$ Discente da Universidade Federal do Delta do Parnaíba, Parnaíba/PI, lucasmsantiago@ hotmail.com

${ }^{3}$ Discente da Universidade Federal do Delta do Parnaíba, Parnaíba/PI, claudia.lima.44@ hotmail.com

${ }^{4}$ Discente da Universidade Federal do Delta do Parnaíba, Parnaíba/PI, iancoandrade@ufpi.edu.br

${ }^{5}$ Mestre em Enfermagem pela Universidade Feder al do Piauí, Teresina-PI, Discente do curso de Medicina da UFDPar, Parnaíba-PI, at.tiago@ hotmail.com
} 


\section{IMPACTO DA PANDEMIA NO RASTREIO DO CÂNCER DE MAMA NO ESTADO DO PIAUÍ.}

Maria Beatriz Pereira de Paula Rocha ${ }^{1}$; Andressa Carvalho Pereira ${ }^{2}$; Vinicius Souza Freires ${ }^{3}$; Rafael Santos Correia ${ }^{4}$; Franciele Basso Fernandes Silva ${ }^{5}$.

Introdução: O câncer de mama é a principal causa de morte por câncer em mulheres no Brasil. A mamografia é o método de escolha para o rastreio dessa neoplasia, sendo fundamental para a sua detecção precoce. $\mathrm{O}$ adiamento ou suspensão desses procedimentos durante a pandemia pode impactar negativamente a assistência oncológica. Objetivo: Analisar o impacto da pandemia sobre o rastreio do câncer de mama no estado do Piauí. Métodos: Estudo epidemiológico com dados secundários obtidos do Sistema de Informação do Câncer (SISCAN), referentes aos exames de rastreio para neoplasia maligna de mama, no estado do Piauí, registrados nos anos de 2019 e 2020 . As variáveis avaliadas foram faixa etária e local de residência. Resultados: Em 2019, foram realizadas 21.936 mamografias no Piauí, já em 2020, essa quantidade reduziu para 17.666. Esse declínio se deu, principalmente, entre os meses de abril a agosto em relação ao ano anterior, sendo maio e junho os que apresentaram maior queda, com uma redução de 96,52\% (64) e 95,11\% (71) de mamografias realizadas, respectivamente. Em contrapartida, em outubro houve um significativo aumento relativo ao ano 2020, com 3.075 mamografias realizadas. A faixa etária de acima de 79 anos apresentou a maior queda no número de mamografias realizadas de um ano para o outro $(-55,55 \%)$. Conclusão: Os dados encontrados sugerem um impacto da pandemia, tais como o distanciamento social e temor da contaminação, sobre a quantidade de mamografias realizadas. $\mathrm{O}$ aumento em outubro pode estar associado às campanhas de prevenção e rastreio, como o Outubro Rosa.

Descritores: Pandemia; Neoplasias da Mama; Mamografia; Epidemiologia.

\footnotetext{
1 Instituto de Educação Superior do Vale do Parnaíba (IESVAP) Parnaíba, Piauí. E-mail: mariabeatriz_rocha@hotmail.com

${ }^{2}$ Universidade Federal do Delta do Parnaíba (UFDPar), Parnaíba, Piauí. E-mail: andressa_carvalho@ufpi.edu.br, https://orcid.org/0000-0001-7721-0661

${ }^{3}$ Universidade Federal do Delta do Parnaíba (UFDPar), Parnaíba, Piauí. E-mail: vinifreires@gmail.com

${ }^{4}$ Universidade Federal do Delta do Parnaíba (UFDPar), Parnaíba, Piauí. E-mail: rafael0094@gmail.com

${ }^{5}$ Universidade Federal do Delta do Parnaíba (UFDPar), Parnaíba, Piauí. E-mail: francibasso2@ hotmail.com
} 


\section{REPOSIÇÃO VOLÊMICA EM PACIENTES VÍTIMAS DE TRAUMA: UMA REVISÃO DE LITERATURA}

Thayná Lacerda Almeida ${ }^{1}$, Evellyn Maria Silva de Almeida ${ }^{2}$, Lohana Maylane Aquino

Correia de Lima ${ }^{3}$, Ricardo Eugenio Varela Ayres de Melo ${ }^{4}$.

Introdução: Uma vítima de trauma pode apresentar hemorragia e evoluir para quadros de choque, devendo os profissionais de saúde estarem preparados para reverter esse cenário a partir da reposição do volume sanguíneo. Objetivo: Objetiva-se expor o protocolo da reposição volêmica e suas indicações em casos de trauma. Métodos: Foi realizada uma revisão de literatura contemplando artigos indexados na PubMed entre 2016 e 2021, que abordassem de forma prática a reposição volêmica. Resultados: De acordo com o protocolo do Advanced Trauma Life Suport, conhecido como o padrão ouro em traumatismos, a terceira medida de atendimento é avaliar as condições circulatórias do paciente, identificando se apresenta má perfusão tecidual, com sinais de taquicardia (frequência cardíaca acima de 100 em adultos) e vasoconstrição cutânea. É observado também a frequência respiratória, o nível de consciência, a pressão de pulso e a diurese do paciente. Se o choque for constatado, é indicado a reposição do volume intravascular perdido se utilizando de soluções eletrolíticas isotônicas aquecidas, como o Ringer lactato ou soro fisiológico. Em adultos, é recomendado de um a dois litros de solução, sendo monitorada a resposta do doente. Em caso de ausência de melhoras à terapia inicial, pode ser necessária a transfusão sanguínea. Conclusão: Desta forma, é fundamental conhecer o protocolo de reposição de fluidos para pacientes vítimas de trauma, a fim de evitar danos aos órgãos e morte decorrente de choque.

Descritores: Hipovolemia; Cuidados de Suporte Avançado de Vida no Trauma; Lactato de Ringer; Choque.

\footnotetext{
${ }^{1}$ Acadêmica em odontologia, Universidade Federal de Pernambuco (UFPE), Recife - PE. thaynalacerda955@gmail.com

${ }^{2}$ Acadêmica em odontologia, Universidade Federal de Pernambuco (UFPE), Recife - PE. evellynmsa@gmail.com.

${ }^{3}$ Mestranda em Clínica Integral pela Universidade Federal de Pernambuco, Recife - PE, lohanawatson@hotmail.com.

${ }^{4}$ Coordenador do curso de Especialização em Cirurgia Buco Maxilo Facial, Universidade Federal de Pernambuco (UFPE), Recife - PE. revamelo@yahoo.com
} 


\title{
PADRÃO ESPACIAL E TEMPORAL DOS ÓBITOS POR AIDS NO ESTADO DO MARANHÃO NO PERÍODO DE 2011 A 2019
}

\author{
Camila da Silva Lopes Nunes ${ }^{1}$, Thatiana Araújo Maranhão
}

Introdução: De 2009 a 2019 o coeficiente de mortalidade pelo vírus da imunodeficiência humana (HIV) e/ou pela Síndrome da Imunodeficiência Adquirida (Aids), caiu 29,3\% no Brasil, mas seis estados caminharam na direção contrária. O Maranhão foi um deles. Em 2009, o estado apresentou um coeficiente de 5,2 por 100 mil habitantes, já em 2019 houve um aumento de 9,6\%. Objetivo: Descrever o perfil epidemiológico e o padrão espaço temporal dos óbitos por aids ocorridos no estado do Maranhão. Métodos: Estudo ecológico em que foram considerados todos os óbitos por aids entre residentes no Maranhão, notificados no Sistema de Informação sobre Mortalidade (SIM), no período de 2011 a 2019. Os dados foram obtidos no sítio eletrônico do Departamento de Informática do SUS, o qual é de domínio público. Para a análise descritiva utilizou-se estatística univariada e foram calculadas taxas de mortalidade, utilizando-se o software TabWin. Resultados: Foram registrados 3.542 óbitos por aids no Maranhão no período analisado. A maioria dos indivíduos era do sexo masculino (2.373; 66,99\%), raça parda $(2.251 ; 65,72 \%)$, faixa etária de 30 a 39 anos $(1.210 ; 34,2 \%)$, escolaridade de 4 a 7 anos de estudo $(916 ; 29,24 \%)$ e estado civil solteiro (2.182; 66,22\%). A taxa de mortalidade média bruta no período foi de 5,7 óbitos por 100 mil habitantes, verificando-se aumento de 18,7\% na mortalidade por aids. Conclusão: Estratégias de intervenção que possibilitem maior acesso a Terapia antirretroviral devem ser estabelecidas a população residente nas regiões de maior incidência de aids, para atenuar as mortes nesses locais.

Descritores: Mortalidade; Síndrome da imunodeficiência adquirida; Análise espaço temporal.

\footnotetext{
${ }^{1}$ Curso de Bacharelado em Enfermagem da Universidade Estadual do Piauí - Campus Professor Alexandre Alves de Oliveira, Parnaíba - Piauí, camilasnunes@aluno.uespi.br, https://orcid.org/0000-0003-1333-5793.

2 Professora Adjunta nível II do curso de Bacharelado em Enfermagem da Universidade Estadual do Piauí Campus Professor Alexandre Alves de Oliveira, Parnaíba - Piauí, thatianamaranhao@phb.uespi.br, https://orcid.org/ 0000-0003-4003-1365.
} 


\title{
REPERCUSSÕES CLÍNICO-OBSTÉTRICAS DE GESTANTES COM COVID-19: UMA REVISÃO SISTEMÁTICA
}

\author{
Sarah Nilkece Mesquita Araújo Nogueira Bastos ${ }^{1}$; Barbara Louise Ferreira Barbosa ${ }^{2}$; Larisse \\ Giselle Barbosa Cruz ${ }^{3}$; Rayza Pereira De Souza ${ }^{4}$ Caroline Camargo Bandeira da Silveira Luz $^{5}$
}

Introdução: O SARS-Cov-2 afeta, especialmente, pacientes em grupos de alto risco. Objetivo: Analisar as repercussões clínico-obstétricas de gestantes com COVID-19. Método: Revisão sistemática nas bases de dados: MEDLINE/PubMed, LILACS, SCIELO e CNKI. Os descritores controlados, utilizados na estratégia de busca foram: "Pregnancy"; "2019-nCov"; "SARS-Cov-2"; "Coronavirus". Elegeram-se títulos originais, sem restrição de idioma e período, e que abordassem gestantes com diagnóstico clínico e/ou laboratorial de COVID-19. Excluíram-se revisões, editoriais,títulos duplicados. Resultados: Foram incluídos 34 artigos, com 412 gestantes infectadas pelo SARS-Cov-2, com idade média de 27,5 anos e média de 36,0 semanas gestacionais. As principais repercussões gestacionais detectadas nos estudos foram: parto cesáreo $(36,2 \% ; 149)$, pneumonia materna grave $(21,6 \% ; 89)$, parto prematuro $(18,4 \% ; 76)$, ruptura prematura membranas ovulares (RPMO) $(3,4 \% ; 14)$, abortamento $(1,0 \% ; 4)$. Foi detectada apenas uma morte materna $(0,2 \% ; 1)$ entre as mulheres analisadas. Conclusão: Gestantes infectadas devem receber uma atenção maior e um cuidado mais abrangente, devido às respostas imunes e a elevada patogenicidade do vírus que ainda permanece obscura.

Descritores: Gravidez; 2019-nCov; SARS-Cov-2; Coronavírus.

\footnotetext{
${ }^{1}$ Universidade Federal do Delta do Parnaíba (UFDPar), Parnaíba, Piauí. E-mail: sarahnilkece@ hotmail.com

${ }^{2}$ Universidade Federal do Delta do Parnaíba (UFDPar), Parnaíba, Piauí. E-mail: barbara._louisee@hotmail.com

${ }^{3}$ Universidade Federal do Delta do Parnaíba (UFDPar), Parnaíba, Piauí. E-mail: larissegiselle@ @otmail.com

${ }^{4}$ Centro Universitário Maurício de Nassau (UNINASSAU), Parnaíba, Piauí. E-mail: rayzapsousa1@gmail.com

${ }^{5}$ Universidade Federal do Delta do Parnaíba (UFDPar), Parnaíba, Piauí. E-mail:

carolinecbsilveira@hotmail.com.br
} 


\title{
A SISTEMÁTICA MULTIDISCIPLINAR NO ATENDIMENTO DE EMERGÊNCIA EM PACIENTES VÍTIMAS DE TRAUMAS FACIAIS.
}

\author{
Shamara Pinto Ferreira da Cruz ${ }^{1}$; Carolina Pereira da Silva ${ }^{2}$; Maria Alice da Silva Souza ${ }^{3}$; \\ Julia Soledade Vieira de Mello ${ }^{4}$; Isaque Ferreira Soares ${ }^{5}$.
}

Introdução: Os traumas que atingem a face são os mais comuns e têm como principais etiologias os acidentes automobilísticos, agressões físicas e quedas da própria altura, podendo ser representados por fraturas ósseas, ferimentos de tecidos moles e traumatismos alvéolodentários, sendo o terço médio a região mais acometida. A alta incidência de lesões faciais leva a uma abordagem multidisciplinar, incluindo desde as primeiras avaliações até os acompanhamentos pós-operatórios. Objetivo: Realizar uma revisão bibliográfica sobre o atendimento sistematizado e multidisciplinar na correta sequência de atendimento para traumas faciais. Métodos: Realizou-se uma revisão literária através do cruzamento dos descritores entre os anos de 2011e 2021, encontrados nos periódicos das bases de dados SCIELO, LILACS, SCOPUS e MEDLINE. Resultados: Entre os tipos de atendimento à vítima do trauma destaca-se o Suporte Avançado de Vida no Trauma, no qual cerca de 25 a $33 \%$ das mortes por trauma podem ser evitadas. Paciente vítima de traumatismo facial pode apresentar lesões de tecidos moles e/ou duros. Sendo assim, deve ser realizado um exame intra e extrabucal e ser avaliado diariamente por todas as equipes envolvidas em seu atendimento. Durante a permanência do paciente no hospital, o cirurgião buco-maxilo-facial deverá proceder à evolução clínica diária do paciente até que ele se encontre em condições de obter alta hospitalar. Conclusão: Desse modo, a comunicação entre os diversos profissionais de saúde envolvidos na equipe torna-se necessária, pois o saber de cada um é imprescindível para que ocorra uma melhora no estado de saúde geral do paciente.

Descritores: Cirurgia Maxilofacial; Cuidados; Equipe multidisciplinar; Traumatismos Maxilofaciais.

\footnotetext{
1 Graduanda em Odontologia pela Universidade de Pernambuco, Recife, PE. E-mail: shamarafdacruz@gmail.com

2 Graduanda em Odontologia pela Universidade de Pernambuco, Recife, PE. E-mail: carolinapereeira@gmail.com

${ }^{3}$ Graduanda em Odontologia pela Universidade de Pernambuco, Recife, PE. E-mail: alice.ssouza@upe.br

${ }^{4}$ Graduanda em Odontologia pela Universidade de Pernambuco, Recife, PE. E-mail: julia.soledade@upe.br

5 Docente do Centro Universitário Facol (UNIFACOL), Vitória de Santo Antão, PE. E-mail: Isaqueodontolab@hotmail.com
} 


\section{UM CONTRAPONTO ENTRE A INTERVENÇÃO CIRÚRGICA E A CONSERVADORA NO TRAUMA HEPÁTICO}

Marcos Lorran Paranhos Leão ${ }^{1}$, Virgínia Alves de Oliveira² ${ }^{2}$ Elaine Cristina Mendes de Oliveira $^{3}$, Luiz Eduardo Correia Miranda ${ }^{4}$.

Introdução: O trauma hepático é fruto da transferência da energia ambiental para o órgão em intensidade maior do que ele suporta, e vai de leve a grave. Pode apresentar desde uma pequena ruptura capsular até danos extensos, com lesão associada à irrigação sanguínea do órgão. Objetivo: Apresentar um contraponto entre a intervenção cirúrgica e a conservadora no tratamento do trauma hepático. Métodos: Trabalho qualitativo, exploratório e bibliográfico integrativo. A busca foi executada nos repositórios: Pubmed, Bireme, Lilacs, Scielo e Google Acadêmico, utilizando os Descritores em Ciências da Saúde: Wounds and injuries "and" Liver "and" Conservative Treatment "or" General Surgery. Resultados: Foram analisados 39 trabalhos. Aproximadamente 8,2\% dos casos de lesão de órgão sólido intra-abdominal (fígado e rim) foram levados para cirurgia e $91,8 \%$ foram tratados com métodos conservadores. A intervenção cirúrgica gerou complicações em 23,7\% dos casos, já a conservadora, em 3 a $10 \%$. Há aumento no uso do tratamento conservador, mesmo em lesões de alto grau, apresentando taxa de eficácia de até $100 \%$ em alguns estudos. Em pacientes hemodinamicamente estáveis, o tratamento não operatório é considerado o padrão-ouro de cuidado. Os traumas de alta energia e ferimentos balísticos geralmente são direcionados ao tratamento operatório. Vários aspectos influenciam na escolha da terapêutica, como o estado hemodinâmico do paciente e a capacidade do serviço de suprir as necessidades que, porventura, se manifestem. Conclusão: Os dois manejos podem ser eficazes se aplicados de maneira correta. Para isso, a escolha da abordagem deve ser pautada em diversos fatores.

Descritores: Ferimentos e lesões. Fígado. Tratamento Conservador. Cirurgia Geral. Traumatismo múltiplo.

\footnotetext{
${ }^{1}$ Faculdade de Ciências Médicas (FCM) da Universidade de Pernambuco (UPE). Recife - Pernambuco. E-mail: marcos.leao@upe.br;

${ }^{2}$ Faculdade de Ciências Médicas (FCM) da Universidade de Pernambuco (UPE). Recife - Pernambuco. E-mail: virginia.alves@upe.br;

${ }^{3}$ Faculdade de Ciências Médicas (FCM) da Universidade de Pernambuco (UPE). Recife - Pernambuco. E-mail: elaine.oliveira@upe.br;

${ }^{4}$ Faculdade de Ciências Médicas (FCM) da Universidade de Pernambuco (UPE). Recife - Pernambuco. E-mail: lecmiranda@gmail.com.
} 


\section{TROMBOEMBOLISMO PULMONAR BILATERAL: RELATO DE CASO DE UM HOSPITAL TERCIÁRIO DE FORTALEZA-CE.}

Ana Beatriz Feijó de Andrade ${ }^{1}$, Bruna Soares Praxedes ${ }^{2}$, Victoria Benigno Moreira da Rocha $^{3}$, Camile Feijó de Andrade ${ }^{4}$, Gabriel Menezes Albuquerque ${ }^{5}$.

Introdução: O tromboembolismo pulmonar (TEP) é uma doença potencialmente fatal e, em conjunto com a trombose venosa profunda, compõe a terceira síndrome cardiovascular aguda mais frequente, o tromboembolismo venoso. Exposição do caso: Mulher, 71 anos, apresentando dor torácica e dispneia há 3 dias. Possui insuficiência cardíaca há um ano e relato de cirurgia ortopédica em membro superior há 5 meses. À admissão, apresentava-se instável hemodinamicamente (pressão arterial 90x60 mmHg e SatO2 91\% em cateter nasal 5L/minuto). Visualizado, em radiografia de tórax, abaulamento do tronco pulmonar e aumento da área cardíaca. Ao ecocardiograma transtorácico, dilatação acentuada de câmaras direitas e do tronco da artéria pulmonar, hipertensão pulmonar importante e disfunção do ventrículo direito. Angiotomografia de tórax evidenciou trombo luminal na artéria pulmonar do lobo superior esquerdo, além de trombo luminal preenchendo a artéria interlobar descendente à direita e a artéria do lobo médio. Diagnosticado tromboembolismo pulmonar bilateral, em uso de anticoagulação plena com enoxaparina. Durante o internamento, evoluiu com hipotensão arterial, tosse produtiva e hemoptise, sendo iniciado dobutamina e nitroglicerina. Devido à piora da dispneia, instabilidade hemodinâmica e ausência de contraindicações, optado por realizar trombólise com Tenecteplase. Evoluiu, posteriormente, com desmame das drogas vasoativas, melhora clínica e hemodinâmica. Conclusão: Dessa forma, é válido ressaltar a importância do diagnóstico precoce no TEP, que deve ser sempre cogitado em pacientes com dispneia, taquipneia, dor torácica ou choque, sendo a instabilidade hemodinâmica uma forma rara, que indica embolia central ou extensa, configurando alto risco de mortalidade. Nesses casos, deve-se proceder para terapia trombolítica sistêmica, assim como foi relatado anteriormente.

Descritores: Embolia Pulmonar; Hipertensão Pulmonar; Emergências.

\footnotetext{
${ }^{1}$ Graduando de Medicina da Universidade de Fortaleza-CE, andradefbeatriz@gmail.com.

${ }^{2}$ Graduando de Medicina da Universidade de Fortaleza-CE, brunaspraxedes@ gmail.com

${ }^{3}$ Graduando de Medicina da Universidade de Fortaleza-CE, victoriabenigno2021@ gmail.com

${ }^{4}$ Graduando de Medicina da Universidade de Fortaleza-CE, camilefeijo21@gmail.com

${ }^{5}$ Médico Cardiologista do Hospital do Coração de Messejana - Dr. Carlos Alberto Studart Gomes, Fortaleza-CE, gabrielmalbuquerque@gmail.com.
} 


\title{
TRATAMENTO CIRÚRGICO DE INVERSÃO UTERINA AGUDA PÓS PARTO: UM ESTUDO COMPARATIVO ENTRE PIAUÍ, NORDESTE E BRASIL.
}

\author{
Ana Vitória Meireles Veiga ${ }^{1}$, Igor dos Santos Cavalcante ${ }^{2}$, Martha Laura Leão dos Santos \\ $\mathrm{Silva}^{3}$, Livia Rocha Santos ${ }^{4}$, Lúcia Maria de Sousa Aguiar dos Santos ${ }^{5}$
}

Introdução: A inversão uterina (IU) é uma emergência médica rara na qual o corpo uterino vira pelo avesso, protraindo-se através do colo do útero até o interior da vagina ou além da abertura vaginal. Objetivo: $\mathrm{O}$ presente trabalho busca fazer um apanhado epidemiológico para o tratamento cirúrgico de IU no Brasil, Nordeste e Piauí no período de 2015 a 2019. Métodos: Trata-se de um estudo epidemiológico, cujos dados foram retirados do SISTEMA DE INFORMAÇÕES HOSPITALARES (SIH/SUS). Os parâmetros utilizados foram local de ocorrência e ano de ocorrência. Resultados: No período analisado foram realizadas 197 intervenções cirúrgicas, sendo 30 (15\%) na região nordeste e 4 casos (2\%) no estado do Piauí. Observou-se que no ano de 2015 foram realizadas 32 intervenções cirúrgicas para tratamento de IU no Brasil, enquanto que em 2016, houveram 26 cirurgias. Em 2017, 40 cirurgias. Em 2018, 42 cirurgias. Em 2019, foram realizadas 57 cirurgias. Discussão: A partir dos dados obtidos, observou-se que o total de procedimentos realizados no país (197), no período estudado, corrobora com a literatura de que essa não é uma intercorrência comum. Além disso, é notável um aumento não linear de cirurgias para tratamento de IU no período, o que pode significar uma melhora no manejo de casos e aprimoramento de técnicas. CONCLUSÃO: É notório que a IU é uma emergência obstétrica que deve ter diagnóstico e terapêutica imediatos devido à alta morbimortalidade materna. É necessária a vigilância atenta da paciente, principalmente no pós-parto, para uma boa recuperação anatômico e funcional, sem sequelas.

Descritores: Inversão Uterina, Período Pós Parto, Hemorragia pós-parto

\footnotetext{
${ }^{1}$ Universidade Federal do Delta do Parnaíba (UFDPar), Parnaíba, Piauí. E-mail: vitmei@ hotmail.com

${ }^{2}$ Universidade Federal do Delta do Parnaíba (UFDPar), Parnaíba, Piauí. E-mail: igorsc@live.com

${ }^{3}$ Universidade Federal do Delta do Parnaíba (UFDPar), Parnaíba, Piauí. E-mail: marthalaura928@gmail.com

${ }^{4}$ Universidade Federal do Delta do Parnaíba (UFDPar), Parnaíba, Piauí. E-mail: liviarocha063@gmail.com

${ }^{5}$ Faculdade Integral Diferencial (FACID), Teresina, Piauí. Email: 1msasantos@gmail.com
} 


\section{TRAUMATISMO DURANTE O PARTO NO NEONATO: UMA ANÁLISE EPIDEMIOLÓGICA COMPARATIVA EM NÍVEL NACIONAL, REGIONAL E ESTADUAL}

Ana Vitória Meireles Veiga ${ }^{1}$, Igor dos Santos Cavalcante ${ }^{2}$, Martha Laura Leão dos Santos Silva $^{3}$, Livia Rocha Santos ${ }^{4}$, Lúcia Maria de Sousa Aguiar dos Santos ${ }^{5}$

Introdução: O parto traumático ocorre devido a algumas lesões resultantes do esforço realizado pela parturiente e pela forma como se deu o processo do parto, o que pode traumatizar fisicamente o recém-nascido. Objetivo: Esse trabalho visa analisar os números de casos de traumatismo no neonato durante o parto em âmbito federal, estadual e regional. Métodos: Trata-se de um trabalho epidemiológico e quantitativo, cujos dados foram retirados do SISTEMAS DE INFORMAÇÕES HOSPITALATES (SIH/SUS). Os parâmetros utilizados foram o período de ocorrência (2015 a 2019) e local de internação. Resultados: No período analisado foram notificados 2305 casos de parto traumático no país, sendo 455 no Nordeste e 18 no Piaui. Em relação ao ano de ocorrência. Ocorreram 509 partos traumáticos em 2015 no Brasil. Em 2016, foram 455 casos. Em 2017, foram 406. Em 2018, foram 498. Em 2019, foram 437. Discussão: Analisando-se os dados obtidos percebese que não houve uma grande variação numérica na quantidade de casos (variação percentual de $14 \%$ entre o primeiro e último ano). Já em comparação com os valores federais, o Nordeste apresenta uma quantidade pequena de casos de aproximadamente $20 \%$, enquanto que o PI possui $0,8 \%$ dos casos registrados. Conclusão: A incidência de lesões neonatais resultante de partos difíceis ou traumáticos está diminuindo, provavelmente, devido ao aumento da realização de cesarianas no país. Contudo, a realização de parto normal deve ser estimulada com a aplicação correta dos protocolos e diretrizes do Ministério da Saúde para que não ocorram traumatismos aos neonatos durante o parto.

Descritores: Recém-nascido, Trabalho de Parto, Complicações do Trabalho de Parto

\footnotetext{
${ }^{1}$ Universidade Federal do Delta do Parnaíba (UFDPar), Parnaíba, Piauí. E-mail: vitmei@ hotmail.com

${ }^{2}$ Universidade Federal do Delta do Parnaíba (UFDPar), Parnaíba, Piauí. E-mail: igorsc@live.com

${ }^{3}$ Universidade Federal do Delta do Parnaíba (UFDPar), Parnaíba, Piauí. E-mail: marthalaura928@gmail.com

${ }^{4}$ Universidade Federal do Delta do Parnaíba (UFDPar), Parnaíba, Piauí. E-mail: liviarocha063@gmail.com

${ }^{5}$ Faculdade Integral Diferencial (FACID), Teresina, Piauí. Email: 1msasantos@gmail.com
} 


\title{
VANTAGENS E DESVANTAGENS DO USO DE ÁCIDO HIALURÔNICO NO PREENCHIMENTO ESTÉTICO DE OLHEIRAS
}

\author{
Anderson Marlon Albino de Abreu ${ }^{1}$; Ana Júlia Gomes Leal²; Nayanne Gomes Araújo³; José \\ Fernando Marinho de Almeida Moreira ${ }^{4}$; Héberte Santana Arruda ${ }^{5}$.
}

Introdução: As olheiras são uma das condições estéticas mais comuns que desagradam muitos indivíduos. Sendo assim, um dos segmentos de expansão mais rápida na indústria médica cosmética para este fim é a utilização de preenchedores de ácido hialurônico. Todavia, o teor de ácido hialurônico na pele diminui com o tempo, devido aos diversos efeitos ambientais e fisiológicos. Objetivos: Analisar as vantagens e desvantagens da utilização do ácido hialurônico no preenchimento de olheiras. Métodos: Revisão simples de literatura realizada nas bases de dados BVS e SciELO, por meio dos termos: Ácido Hialurônico; Face; Preenchedores Dérmicos. Após aplicação dos critérios de inclusão e exclusão, foram selecionados 10 artigos que constam nessa revisão. Resultados: O ácido hialurônico apresenta várias vantagens, pois ele consegue se integrar aos tecidos, permitindo a passagem do oxigênio para as células mantendo-as hidratadas, com efeito de maciez e naturalidade. Além disso, encontra-se presente nas camadas basais da pele, não causando assim reação inflamatória e sua aplicação é quase que indolor, devido à utilização de anestesia local no procedimento de preenchimento. Seu uso ajuda na reparação tecidual, estímulo de colágeno, além de proteger a pele contra fatores intrínsecos e extrínsecos. As maiores desvantagens são a baixa durabilidade dos resultados, que são revelados de 6 a 12 meses após o uso e seu o custo elevado. Conclusão: Portanto, o uso de ácido hialurônico injetável vem conquistando lugar de destaque no complexo processo de envelhecimento infraorbital, proporcionando uma harmonização orofacial, melhorando assim o bem estar físico, mental, e social do paciente.

Descritores: Ácido Hialurônico; Face; Preenchedores Dérmicos.

\footnotetext{
${ }^{1}$ Universidade Federal de Pernambuco - UFPE - Recife-PE, Graduando de Odontologia, $6^{\circ}$ período, andersonabreu2012@gmail.com

${ }^{2}$ UFPE - Recife-PE, Graduanda de Odontologia, $6^{\circ}$ período, lealgomesjulia@ hotmail.com

${ }^{3}$ UFPE - Recife-PE, Graduanda de Odontologia, 6 período, gomesnayanne7@gmail.com

${ }^{4}$ UFPE - Recife-PE, Graduanda de Odontologia, $6^{\circ}$ período, fernandinhobrawli@ hotmail.com

${ }^{5}$ Faculdade de Odontologia de Pernambuco UPE- Recife-PE, Prof. MSc do Curso de Odontologia do Centro

Universitário Brasileiro - Unibra, hebertearruda@gmail.com
} 